\title{
Invariant tori of full dimension for higher-dimensional beam equations with almost-periodic forcing
}

Jie Rui ${ }^{*}$ and Yi Wang ${ }^{2}$

"Correspondence: rjhygl@163.com
'College of Science, China
University of Petroleum, Qingdao,
People's Republic of China
Full list of author information is
available at the end of the article

\begin{abstract}
In this paper, we focus on the class of almost-periodically forced higher-dimensional beam equations

$$
u_{t t}+(-\Delta+\mu)^{2} u+\psi(\omega t) u=0, \quad \mu>0, t \in \mathbb{R}, x \in \mathbb{R}^{d}
$$

subject to periodic boundary conditions, where $\psi(\omega t)$ is real analytic and almost-periodic in $t$. We show the existence of almost-periodic solutions for this equation under some suitable hypotheses. In the proof, we improve the KAM iteration to deal with the infinite-dimensional frequency $\omega=\left(\omega_{1}, \omega_{2}, \ldots\right)$.
\end{abstract}

MSC: $37 \mathrm{~K} 55 ; 11 \mathrm{~K} 70$

Keywords: Infinite-dimensional Hamiltonian systems; Beam equations; Almost-periodic solutions; KAM theory

\section{Introduction}

Recently, many researchers focus on some physical models appeared in dynamics of the suspension bridge, nonrelativistic quantum mechanics, supersymmetric field theoriesk and inflation cosmology [1-11]. For those models, there are many remarkable results on the global existence or the blowup of solutions for wave equations [2-4], elliptic equations [5, 6], and some semilinear evolution equations [7]. As in the fundamental models, the dynamical behavior of the solutions is studied. The decay estimate of the solution at both subcritical and critical initial energy levels was obtained by Xu [2]. Nguyen [8] considered the compactness and stability for the Maxwell equations. Goubet and Manoubi [9] investigated the asymptotic convergence of the solutions.

For one-dimensional Hamiltonian systems, the existence of quasiperiodic solutions or almost-periodic solutions is also very significant in physics. It is well known that the infinite-dimensional KAM theory is powerful to obtain it (see [12-21]). However, the standard KAM theory fails to study higher-dimensional Hamiltonian PDEs because of the multiplicity of the eigenvalues.

(c) The Author(s) 2020. This article is licensed under a Creative Commons Attribution 4.0 International License, which permits use, sharing, adaptation, distribution and reproduction in any medium or format, as long as you give appropriate credit to the original author(s) and the source, provide a link to the Creative Commons licence, and indicate if changes were made. The images or other third party material in this article are included in the article's Creative Commons licence, unless indicated otherwise in a credit line to the material. If material is not included in the article's Creative Commons licence and your intended use is not permitted by statutory regulation or exceeds the permitted use, you will need to obtain permission directly from the copyright holder. To view a copy of this licence, visit http://creativecommons.org/licenses/by/4.0/. 
It is worth noting that the first breakthrough in higher-dimensional PDEs is due to Bourgain. Bourgain [22] obtained quasiperiodic solutions for two-dimensional nonlinear Schrödinger equations via the developed Craig-Wayne methods. The Craig-WayneBourgain methods can overcome the difficulty of the asymptotical multiplicity of eigenvalues in higher-dimensional PDEs. However, it should also be pointed out that the KAM theory has some important advantages. We can construct a local normal form in a neighborhood of the solutions using the KAM theory, which turns out the behavior and dynamics of the equation of motion. Thereafter, the infinite KAM theorem was extended to the existence of finite-dimensional tori for higher-dimensional Hamiltonian systems. Geng and You [23, 24] constructed KAM theorems for the higher-dimensional beam equation. Yuan [25] obtained a KAM theorem to apply to partial differential equations of higher dimensions.

However, there is a crucial condition in the KAM theorems in [23] and [24] that the nonlinearity $f(u)$ does not explicitly contain the time variable $t$ and the space variable $x$. Thus their KAM approaches failed in the case of the nonlinearity depending on $t$ or $x$. Physically, it requires no external force acting when the string is at rest, tending to distort its equilibrium of $u=0$. Up to now there are very few results on the reducibility in higher dimensions. Eliasson and Kuksin [26] (also see [27]) showed the reducibility for the linear Schrödinger equations in higher dimension

$$
\dot{u}=-\mathrm{i}\left(\Delta u-\epsilon V\left(\phi_{0}+t \omega, x ; \omega\right) u\right), \quad x \in \mathbb{T}^{d} .
$$

Eliasson, Grébert, and Kuksin [28] also considered the $d$-dimensional beam equation, which is a good model for the Klein-Gordon equation. Rui and Liu [29] proved the existence of quasiperiodic solutions for a linear $d$-dimensional beam equation with a quasiperiodic in time potential.

Comparing with the case of quasiperiodic solutions in higher dimensions, as far as we know, the reducibility results for almost-periodic solutions in higher dimensions have not been previously regarded in the literature. In this paper, we focus on the reducibility of the linear $d$-dimensional beam equation with almost-periodic forcing

$$
u_{t t}+(-\Delta+\mu)^{2} u+\psi(\omega t) u=0, \quad \mu>0, t \in \mathbb{R}, x \in \mathbb{R}^{d},
$$

with periodic boundary conditions

$$
u\left(t, x_{1}+2 \pi, \ldots, x_{d}\right)=\cdots=u\left(t, x_{1}, \ldots, x_{d}+2 \pi\right)=u\left(t, x_{1}, \ldots, x_{d}\right),
$$

where $\psi(\omega t)$ is real analytic and almost-periodic in $t$. Our aim is to construct almostperiodic solutions of small amplitude for the beam equation (1.1). This equation is an important model of mathematical physics. It is of great interest in applying to many engineering fields.

Our beam equation (1.1) is quite different from the equations mentioned. There is almost-periodic forcing in higher dimensions, because the reducibility is complex and doubtful. Unfortunately, all those KAM theorems fail to handle infinite-dimensional frequency $\omega=\left(\omega_{1}, \omega_{2}, \ldots\right)$ in Eq. (1.1). Using the method of Pöschel [19] and Xu and You [30], we succeed in decomposing infinite-dimensional frequency in the reducibility. Our 
nonresonance condition of an infinite-dimensional frequency benefits a lot from Pöschel [14]. The main difficulty in this problem is estimating measures of small divisors, since the infinite-dimensional frequency will handle at each step of the KAM iteration. The KAM theory in Kuksin [12] and Pöschel [13] cannot be directly applied to the $d$-dimensional beam equation with almost-periodic forcing, and we will improve the KAM iteration (see Sect. 3). A new strategy to overcome the difficulty are the techniques of decomposing infinitely many frequencies and expanding Hamiltonian into proper series, which are the main achievements of this paper. The author of this paper in [31] and [32] obtained the existence of almost-periodic solutions with almost-periodic forcing using similar techniques. However, Eq. (1.1) is a higher-dimensional equation, and therefore the analysis of Birkhoff normal forms and more precise estimation of new perturbation is very difficult because of the effects of infinite-dimensional frequency,

To state the main results of our paper, we need the following assumptions and sets. To dispose the infinite-dimensional frequency, we construct a sequence $\left\{b_{v}\right\}_{v \geq 0}$ satisfying $b_{0}=2 b \geq 2, b_{v+1}>b_{v}$, and $b_{v} \in \mathbb{Z}^{+}$. We choose the index set

$$
\mathcal{I}_{v}=\left\{i_{j}: j \leq b_{v}, j, i_{j} \in \mathbb{Z}^{+}\right\}, \quad v=0,1, \ldots
$$

The frequencies can be split up as $\omega=\left(\omega^{b_{\nu}}, \omega_{\nu}^{\prime}\right)=\left(\omega_{i_{1}}, \ldots, \omega_{i_{b_{\nu}}}, \omega_{i_{b_{\nu}+1}}, \ldots\right)$. Let $\theta=\omega t$. For fixed $\varrho \in(0,1)$, by [14] the frequency $\omega$ satisfies the following nonresonance conditions

$$
\begin{aligned}
\mathcal{O}:= & \left\{\omega=\left(\omega^{b_{\nu}}, \omega_{\nu}^{\prime}\right) \in \mathcal{O}^{v} \times \mathcal{O}_{\nu}^{\prime} \in[\varrho, 2 \varrho]^{\mathbb{Z}^{+}}:\right. \\
& \left.|\langle k, \omega\rangle| \geq \frac{\tilde{\alpha}}{\exp \left(4|k|^{\tilde{\alpha}} / \tilde{\alpha}\right)}, k \in \mathbb{Z}^{\infty} \backslash\{0\}\right\},
\end{aligned}
$$

where $0<\tilde{\alpha}<1$ is arbitrary and fixed, $|k|=\sum_{i=1}^{\infty}\left|k_{i}\right|, \mathcal{O}^{v}$ is a closed set in $\mathbb{R}^{b_{\nu}}$, and $\mathcal{O}_{v}^{\prime}$ is a closed set. The frequencies $\omega^{b_{\nu}}$ will be chosen properly by the KAM iteration. We also need the following notation:

$$
\begin{array}{ll}
\theta^{b_{\nu}}=\left(\theta_{i_{1}}, \ldots, \theta_{i_{b_{\nu}}}\right), & \theta_{1}^{b_{0}}=\left(\theta_{i_{1}}, \ldots, \theta_{i_{b_{0}}}\right), \quad \theta_{1}^{b_{\nu+1}}=\left(\theta_{i_{b_{v}+1}}, \ldots, \theta_{i_{b_{v+1}}}\right), \\
\omega_{1}^{b_{0}}=\left(\omega_{i_{1}}, \ldots, \omega_{i_{b_{0}}}\right), & \omega_{1}^{b_{\nu+1}}=\left(\omega_{i_{b_{v}+1}}, \ldots, \omega_{i_{b_{v+1}}}\right), \quad v=0,1, \ldots
\end{array}
$$

To apply the KAM theory, we introduce the following assumptions:

(H1) The function $\psi(\omega t)$ is real analytic and almost-periodic with $\omega \in \mathcal{O}$.

(H2) The function $\psi(\theta)$ has a special series expansion of the form

$$
\psi(\theta)=\sum_{j=0}^{\infty} \varepsilon^{b_{j}} \psi_{b_{j}}\left(\theta_{1}^{b_{j}}\right)
$$

which is absolutely convergent. There exists an absolute constant $C$ such that

$$
\begin{aligned}
& |\psi(\theta)| \leq C, \quad\left|\psi_{b_{j}}\left(\theta_{1}^{b_{j}}\right)\right| \leq C, \quad\left|\partial_{\theta_{j}} \psi_{b_{0}}\left(\theta_{1}^{b_{0}}\right)\right| \leq C, \quad j \in \mathcal{I}_{0}, \\
& \left|\partial_{\theta_{j}} \psi_{b_{j}}\left(\theta_{1}^{b_{j}}\right)\right| \leq C, \quad j \in \mathcal{I}_{j} \backslash \mathcal{I}_{j-1}, j=1, \ldots
\end{aligned}
$$


By assumption (H1) we can expand $\psi_{b_{j}}\left(\theta_{1}^{b_{j}}\right)(j=0,1, \ldots)$ into the converging FourierTaylor series

$$
\psi_{b_{0}}\left(\theta_{1}^{b_{0}}\right)=\sum_{k \in \mathbb{Z}^{b_{0}}} \bar{\psi}_{k}^{b_{0}} e^{\mathrm{i}\left\langle k, \theta_{1}^{b_{0}}\right\rangle}, \quad \psi_{b_{j}}\left(\theta_{1}^{b_{j}}\right)=\sum_{k \in \mathbb{Z}^{b_{j}-b_{j-1}}} \bar{\psi}_{k}^{b_{j}} e^{\mathrm{i}\left\langle k, \theta_{1}^{b_{j}}\right\rangle}, \quad j=1, \ldots
$$

Theorem 1.1 (Main Theorem) Assume that $\psi(\theta)$ in (1.1) satisfies assumptions (H1) and (H2). For $\omega=\left(\omega_{1}, \ldots, \omega_{j}, \ldots\right)_{j \in \mathbb{Z}} \in \mathcal{O}$, there exists $\varepsilon$ small enough and a positive Lebesgue measure set $\mathcal{O}^{*} \subset \mathcal{O}$ such that meas $\left(\mathcal{O} \backslash \mathcal{O}^{*}\right) \leq C \varepsilon^{\frac{1}{6}}$. Moreover, for all $\omega^{*}=\left(\omega_{i_{1}}, \ldots\right)_{i_{j} \in \mathcal{I}_{\infty}} \in$ $\mathcal{O}^{*}$, the higher-dimensional beam equation (1.1) with periodic boundary conditions (1.2) admits a family of almost periodic solutions of the form

$$
u(t, x)=\sum_{k \in \mathbb{Z}_{*}^{\infty}} u_{k}(x) e^{\mathrm{i}\left(k, \lambda^{*}\right) t},
$$

where $\lambda^{*}=\left(\cdots, \lambda_{n \infty}, \ldots\right)_{n \in \mathbb{Z}^{d}}$ and $\lambda_{n \infty}=|n|^{2}+\mu+\varepsilon^{b_{0}} \frac{\bar{\psi}_{0}^{b_{0}}}{2 \lambda_{n}}+O\left(\varepsilon^{b_{1}}\right)$.

Remark 1.2 Assumption (H2) is crucial to have a successful KAM iteration. We will split the Hamiltonian and add some proper parts of perturbations to increase the number of frequencies in the next KAM step. Moreover, for the reducibility, we need to ensure that the new perturbation in the next KAM step is smaller than the previous one. Thus $\psi(\theta)$ needs a special series expansion, and the form of the series is decided by KAM iteration.

Remark 1.3 The function $\psi(\theta)$ in (1.1) depends only on $t$ to conserve the partial zeromomentum property in the KAM iteration. Otherwise, in the case of higher dimension the estimate of the new perturbations becomes doubtful, and the terms of new normal form cannot be handled. It is harder than one-dimensional equations in [31] and [32].

The rest of the paper is organized as follows. In Sect. 2, we discuss the Hamiltonian setting corresponding Eq. (1.1). Section 3 is devoted to the reducibility of proving the existence of almost-periodic solutions for the linear $d$-dimensional beam equation using an improved KAM iteration. The small divisors estimate in reducibility is given in the Appendix.

\section{The Hamiltonian of the higher-dimensional beam equation}

In this section, we analyze the Hamiltonian of the higher-dimensional beam equation, which will be transformed into the KAM iteration.

We first introduce some notations. Let $l^{a, \rho}$ be the Banach spaces of complex-valued sequences $q=\left(\cdots, q_{n}, \ldots\right)_{n \in \mathbb{Z}^{d}}$ and its complex conjugate $\bar{q}=\left(\cdots, \bar{q}_{n}, \ldots\right)_{n \in \mathbb{Z}^{d}}$ with the weighted norm

$$
\|q\|_{a, \rho}=\sum_{n \in \mathbb{Z}^{d}}\left|q_{n}\right||n|^{a} e^{|n| \rho}
$$

where $a \geq 0, \rho>0,|n|=\sqrt{n_{1}^{2}+\cdots, n_{d}^{2}}, n=\left(n_{1}, \ldots, n_{d}\right)$. Let $\alpha \equiv\left(\cdots, \alpha_{n}, \ldots\right)_{n \in \mathbb{Z}^{d}}, \beta \equiv$ $\left(\cdots, \beta_{n}, \ldots\right)_{n \in \mathbb{Z}^{d}}, \alpha_{n}, \beta_{n} \in \mathbb{N}$, with finitely many nonzero components of positive integers. The product $q^{\alpha} \bar{q}^{\beta}$ denotes $\Pi_{n} q_{n}^{\alpha_{n}} \bar{q}_{n}^{\beta_{n}}$. 
In the following, we reduce the Hamiltonian of the higher-dimensional beam equation (1.1). The Hamilton systems (1.1)-(1.2) are equivalent to the systems

$$
u_{t}=v, \quad v_{t}=-A^{2} u-\psi(\omega t) u, \quad A u=(-\Delta+\mu) u .
$$

By a simple computation, $\lambda_{n}=|n|^{2}+\mu$ are the eigenvalues of the operator $A=-\Delta+\mu$ subject to periodic boundary conditions with eigenfunctions $\phi_{n}(x)=\sqrt{\frac{1}{(2 \pi)^{d}}} e^{\mathrm{i}\langle n, x\rangle}, n \in \mathbb{Z}^{d}$. Letting $q=\frac{1}{\sqrt{2}} A^{\frac{1}{2}} u-\mathrm{i} \frac{1}{\sqrt{2}} A^{-\frac{1}{2}} \nu$, we have

$$
q_{t}=\mathrm{i}\left(A q+\frac{1}{\sqrt{2}} \psi(\omega t) A^{-1}\left(\frac{q+\bar{q}}{\sqrt{2}}\right)\right)
$$

Let $q(x)=\sum_{n \in \mathbb{Z}^{d}} q_{n} \phi_{n}(x)$. Equation (2.1) is equivalent to the nonautonomous lattice Hamiltonian system

$$
\dot{q}_{n}=\mathrm{i}\left(\lambda_{n} q_{n}+\frac{\partial G}{\partial \bar{q}_{n}}\right), \quad G(q, \bar{q})=\frac{1}{2} \psi(\omega t) \int_{\mathbb{T}^{d}}\left(\sum_{n \in \mathbb{Z}^{d}} \frac{q_{n} \phi_{n}+\bar{q}_{n} \bar{\phi}_{n}}{\sqrt{2 \lambda_{n}}}\right)^{2} d x
$$

with the corresponding Hamiltonian function

$$
H=\sum_{n \in \mathbb{Z}^{d}} \lambda_{n} q_{n} \bar{q}_{n}+\frac{1}{2} \psi(\omega t) \int_{\mathbb{T}^{d}}\left(\sum_{n \in \mathbb{Z}^{d}} \frac{q_{n} \phi_{n}+\bar{q}_{n} \bar{\phi}_{n}}{\sqrt{2 \lambda_{n}}}\right)^{2} d x
$$

Letting $\theta=\omega t$, we introduce a pair of action-angle variables $(J, \theta) \in \mathbb{R}^{\infty} \times \mathbb{T}^{\infty}$ with

$$
\dot{\theta}=\omega, \quad \dot{J}=-\frac{\partial H}{\partial \theta}, \quad \dot{q}_{n}=\mathrm{i} \frac{\partial H}{\partial \bar{q}_{n}}, \quad n \in \mathbb{Z}^{d} .
$$

Thus the corresponding Hamiltonian function of system (2.2) may be rewritten as

$$
H=\langle\omega, J\rangle+\sum_{n \in \mathbb{Z}^{d}} \lambda_{n} q_{n} \bar{q}_{n}+\frac{1}{2} \psi(\theta) \int_{\mathbb{T}^{d}}\left(\sum_{n \in \mathbb{Z}^{d}} \frac{q_{n} \phi_{n}+\bar{q}_{n} \bar{\phi}_{n}}{\sqrt{2 \lambda_{n}}}\right)^{2} d x
$$

with the symplectic structure $d J \wedge d \theta+\mathrm{i} \sum_{n \in \mathbb{Z}^{d}} d q_{n} \wedge d \bar{q}_{n}$.

By Assumption (H2) the Hamiltonian (2.4) can be split into the following form

$$
\begin{aligned}
& H=\Lambda+P=H_{0}^{1}+H_{1}+\cdots+H_{j}+\cdots \\
& =\left(\Lambda_{0}+R_{0}\right)+\left(\Lambda_{1}+R_{1}\right)+\cdots+\left(\Lambda_{j}+R_{j}\right)+\cdots,
\end{aligned}
$$

where for $j=1, \ldots$,

$$
\begin{aligned}
& H_{0}^{1}=\Lambda_{0}+R_{0}=\left\langle\omega^{0}, J^{0}\right\rangle+\sum_{n \in \mathbb{Z}^{d}} \lambda_{n} q_{n} \bar{q}_{n} \\
&+\frac{1}{2} \varepsilon^{b_{0}} \psi_{b_{0}}\left(\theta_{1}^{b_{0}}\right) \int_{\mathbb{T}^{d}}\left(\sum_{n \in \mathbb{Z}^{d}} \frac{q_{n} \phi_{n}+\bar{q}_{n} \bar{\phi}_{n}}{\sqrt{2 \lambda_{n}}}\right)^{2} d x \\
& H_{j}=\Lambda_{j}+R_{j}=\left\langle\omega_{1}^{b_{j}}, J_{1}^{b_{j}}\right\rangle+\frac{1}{2} \varepsilon^{b_{j}} \psi_{b_{j}}\left(\theta_{1}^{b_{j}}\right) \int_{\mathbb{T}^{d}}\left(\sum_{n \in \mathbb{Z}^{d}} \frac{q_{n} \phi_{n}+\bar{q}_{n} \bar{\phi}_{n}}{\sqrt{2 \lambda_{n}}}\right)^{2} d x .
\end{aligned}
$$


Furthermore, for $j=0,1, \ldots, R_{j}$ may be rewritten in detail as follows:

$$
R_{j}=\sum_{n, m \in \mathbb{Z}^{d}}\left(R_{j n m}^{20 b_{j}} q_{n} q_{m}+R_{j n m}^{11 b_{j}} q_{n} \bar{q}_{m}+R_{j n m}^{02 b_{j}} \bar{q}_{n} \bar{q}_{m}\right)
$$

with

$$
\begin{aligned}
& R_{j n m}^{11 b_{j}}=\frac{\varepsilon^{b_{j}} \psi_{b_{j}}\left(\theta_{1}^{b_{j}}\right)}{2 \sqrt{\lambda_{n} \lambda_{m}}} \int_{\mathbb{T}^{d}} \phi_{n} \bar{\phi}_{m} d x, \quad R_{j n m}^{20 b_{j}}=\frac{\varepsilon^{b_{j}} \psi_{b_{j}}\left(\theta_{1}^{b_{j}}\right)}{4 \sqrt{\lambda_{n} \lambda_{m}}} \int_{\mathbb{T}^{d}} \phi_{n} \phi_{m} d x, \\
& R_{j n m}^{02 b_{j}}=\frac{\varepsilon^{b_{j}} \psi_{b_{j}}\left(\theta_{1}^{b_{j}}\right)}{4 \sqrt{\lambda_{n} \lambda_{m}}} \int_{\mathbb{T}^{d}} \bar{\phi}_{n} \bar{\phi}_{m} d x .
\end{aligned}
$$

Let $b_{-1}=0$. By $q(x)=\sum_{n \in \mathbb{Z}^{d}} q_{n} \phi_{n}(x)$ we can rewrite $R_{j}\left(\theta_{1}^{b_{j}}, q, \bar{q}\right), j=0,1, \ldots$, as follows:

$$
R_{j}\left(\theta_{1}^{b_{j}}, q, \bar{q}\right)=\sum_{\alpha, \beta} R_{j \alpha \beta}(\theta) q^{\alpha} \bar{q}^{\beta}=\sum_{\alpha, \beta} \sum_{k \in \mathbb{Z}^{b_{j}-b_{j-1}}} \frac{1}{\sqrt{\lambda^{\alpha+\beta}}} R_{j k \alpha \beta} e^{\mathrm{i}\left(k, \theta_{1}^{b_{j}}\right\rangle} q^{\alpha} \bar{q}^{\beta},
$$

where $\lambda=\left(\cdots, \lambda_{n}, \ldots\right)_{n \in \mathbb{Z}^{d}}$. It is easy to see that $R_{j}\left(\theta_{1}^{b_{j}}, q, \bar{q}\right), j=0,1, \ldots$, admit the partial zero-momentum property

$$
R_{j k \alpha \beta}=0 \quad \text { whenever } \sum_{n \in \mathbb{Z}^{d}}\left(\alpha_{n}-\beta_{n}\right) n \neq 0 .
$$

Remark 2.1 Property of (2.9) is important for higher-dimensional Hamiltonian systems. It ensures the form of perturbations and the obtained normal form in the KAM iteration. There is a crucial difference from the one-dimensional case. Thus, to conserve this property at each KAM step, we require that $\psi$ does not explicitly depend on space variable $x$.

Remark 2.2 By (2.9) we get $R_{j k n m}^{20 b_{j}}=R_{j k \alpha \beta}, \alpha=e_{n}+e_{m}, \beta=0 ; R_{j k n m}^{02 b_{j}}=R_{j k \alpha \beta}, \alpha=0, \beta=e_{n}+e_{m}$; and $R_{j k n m}^{11 b_{j}}=R_{j k \alpha \beta}, \alpha=e_{n}, \beta=e_{m}$. The perturbation $R_{j}\left(\theta_{1}^{b_{j}}, q, \bar{q}\right)$ satisfying (2.9) means that $R_{j k n m}^{11 b_{j}}=0$ if $n \neq m$. Then the normal variables $q_{n}, \bar{q}_{m}$ with $n \neq m$ in the new normal form will not be coupled. Moreover, there are no terms of the forms $\sum_{n} R_{j k\left(e_{n}+e_{-n}\right) 0} q_{n} q_{-n}$ and $\sum_{n} R_{j k 0\left(e_{n}+e_{-n}\right)} \bar{q}_{n} \bar{q}_{-n}$.

Lemma 2.3 For $a \geq 0$ and $\rho>0$, the gradient $R_{\bar{q}}$ is a real analytic map from a neighborhood of the origin of $l^{a, \rho}$ into $l^{a+1, \rho}$, with

$$
\left\|X_{R}\right\|_{\bar{a}, \rho}=O\left(\|q\|_{a, \rho}\right), \quad \bar{a}=a+1 .
$$

For the proof of Lemma 2.3, see [33].

Remark 2.4 We require that $\bar{a}>a$, which means that the weight of vector fields is a little heavier than that of $q, \bar{q}$. The regularity of $\left\|X_{R}\right\|_{\bar{a}, \rho}$ ensures that $X_{R}$ sends a decaying $q$ sequence to a faster decaying sequence. 


\section{Reducibility}

In this section, we state the important Theorem 3.2 and give a detailed proof of the reducibility to obtain the Theorem 1.1. The main program of proof comes form the KAM iteration, which involves an infinite sequence of change of variables. Thus, at each step of KAM iteration the estimates of the coordinate transformation and the Lebesgue measure of a small devisor are necessary (see Sects. 3.2-3.4). Because of our infinite frequencies in beam equations, we need to improve the program of the KAM iteration (see Sect. 3.5). Since at each KAM step the perturbation must become more smaller than at the previous KAM step, we estimate the new perturbation (see Sect. 3.6). For a high-dimensional beam equation, we need to verify the partial zero-momentum property at each KAM step (see Sect. 3.7). The normal form is obtained by the infinite transforms. Thus the convergence of the infinite transforms needs to be considered (see Sect. 3.8).

\subsection{A theorem of reducibility}

We further give a theorem of reducibility for the Hamiltonian systems (2.5). To this end, we firstly introduce some notations and spaces.

We choose a proper sequence $\left\{b_{v}\right\}_{v=0}^{\infty}$ defined by $b_{0}=2 b>2, b_{v+1}=b_{v}+b=(v+3) b, b_{v} \in$ $\mathbb{Z}^{+}$. Let $\varepsilon_{v}=\varepsilon^{b_{\nu}}, v=0,1, \ldots$ For given $\sigma>0$ and $r>0$, we define the sequences $\left\{\sigma_{v}\right\}_{v=0}^{\infty}$ and $\left\{r_{\nu}\right\}_{v=0}^{\infty}$, as follows:

$$
\begin{aligned}
& \sigma_{0}=\sigma, \quad \sigma_{v}=\sigma_{0}\left(1-\frac{\sum_{i=1}^{v} i^{-2}}{2 \sum_{i=1}^{\infty} i^{-2}}\right), \\
& r_{0}=r, \quad r_{v+1}=\frac{1}{4} \varepsilon^{\frac{1}{8} v b+\frac{1}{12} b}\left(\left(\frac{256\left(b_{v}+4\right)}{e}\right)^{b_{v}+4}\left(\sigma_{v}-\sigma_{v+1}\right)^{-2 b_{v}-\frac{7}{2}}\right)^{\frac{1}{3}} r_{v} .
\end{aligned}
$$

We easily to see that $\sigma_{0}>\cdots>\sigma_{v}>\sigma_{v+1}>\cdots>\sigma / 2$. Denote

$$
D_{v}=D\left(\sigma_{v}, r_{v}\right)=\left\{(\theta, J, q, \bar{q}):|\operatorname{Im} \theta|<\sigma_{v},|J|<r_{v}^{2},\|q\|_{a, \rho}<r_{\nu},\|\bar{q}\|_{a, \rho}<r_{\nu}\right\}
$$

where $|\cdot|$ denotes the sup-norm for complex vectors or matrixes. For $v=0,1, \ldots$, we define that

$$
\begin{aligned}
& \Theta_{\nu}=\left\{\theta: \theta:=\left(\theta^{b_{\nu}}, \theta^{\prime}\right):=\left(\theta_{i_{1}}, \ldots, \theta_{i_{b_{v}}}, \theta_{i_{b_{v}+1}}, \ldots\right),\left|\operatorname{Im} \theta_{j}\right|<\sigma_{\nu}, j=i_{1}, \ldots, i_{b_{v}}, i_{b_{v}+1}, \ldots\right\}, \\
& \mathcal{O}_{\nu}=\left\{\omega: \omega:=\left(\omega^{b_{\nu}}, \omega^{\prime}\right):=\left(\omega_{i_{1}}, \ldots, \omega_{i_{b_{v}}}, \omega_{i_{b_{v}+1}}, \ldots\right) \in \mathcal{O}^{b_{\nu}} \times \mathcal{O}_{\nu}^{\prime}, i_{j} \in \mathcal{I}_{\nu}\right\}, \\
& J^{b_{v}}=\left(J_{i_{1}}, \ldots, J_{i_{b_{v}}}\right), J_{1}^{b_{0}}=\left(J_{i_{1}}, \ldots, J_{i_{b_{0}}}\right), \quad J_{1}^{b_{v+1}}=\left(J_{i_{\left(b_{v}+1\right)}}, J_{i_{\left(b_{v}+2\right)}}, \ldots, J_{i_{(v+1)}}\right) \text {. }
\end{aligned}
$$

We assume that the given analytic function has the following form:

$$
F(\theta, J, q, \bar{q})=\sum_{\alpha, \beta} F_{\alpha \beta}(\theta, J) q^{\alpha} \bar{q}^{\beta}
$$

with the weighted norm

$$
\|F\|_{D(\sigma, r), \mathcal{O}}=\sup _{\|q\|_{a, \rho}<r,\|\bar{q}\|_{a, \rho}<r} \sum_{\alpha, \beta}\left\|F_{\alpha \beta}\right\|_{\Theta \times \mathcal{O}}\left|q^{\alpha} \| \bar{q}^{\beta}\right|
$$


where

$$
F_{\alpha \beta}=\sum_{k \in \mathbb{Z}, \hat{l} \in \mathbb{N}} F_{k \hat{l} \alpha \beta} J^{\hat{l}} e^{\mathrm{i}<k, \theta>}
$$

and

$$
\begin{aligned}
\left\|F_{\alpha \beta}\right\|_{\Theta \times \mathcal{O}} & \equiv \sum_{k, \hat{l}}\left|F_{k \hat{l} \alpha \beta}(\omega)\right|_{\mathcal{O}} r^{2|\hat{l}|} e^{|k| \sigma}, \\
\left|F_{k \hat{l} \alpha \beta}(\omega)\right|_{\mathcal{O}} & =\sup _{\omega \in \mathcal{O}}\left(\left|F_{k \hat{l} \alpha \beta}\right|+\left|\frac{\partial F_{k \hat{k} \alpha \beta}}{\partial \omega}\right|\right) .
\end{aligned}
$$

Let $w^{*}=(\theta, J, q, \bar{q}) \in D(\sigma, r)$. The weighted norm of $w^{*}$ is

$$
\left|w^{*}\right|_{D(\sigma, r)}=|\theta|+\frac{1}{r^{2}}|J|+\frac{1}{r}\|q\|_{a, \rho}+\frac{1}{r}\|\bar{q}\|_{a, \rho} .
$$

For $B(\eta ; \omega): D(\sigma, r) \rightarrow D(\sigma, r)$ and $(\eta ; \omega) \in D(\sigma, r) \times \mathcal{O}$, we denote the operator norms

$$
\begin{aligned}
|B(\eta ; \omega)|_{D(\sigma, r) \times \mathcal{O}} & =\sup _{(\eta ; \omega) \in D(\sigma, r) \times \mathcal{O}} \sup _{w^{*} \neq 0} \frac{\left|B(\eta ; \omega) w^{*}\right|_{a, \rho}}{\left|w^{*}\right|_{\rho}}, \\
|B(\eta ; \omega)|_{D(\sigma, r) \times \mathcal{O}}^{*} & =\max \left\{|B(\eta ; \omega)|_{D(\sigma, r) \times \mathcal{O}},\left|\partial_{\omega} B(\eta ; \omega)\right|_{D(\sigma, r) \times \mathcal{O}}\right\} .
\end{aligned}
$$

For the Hamiltonian vector field $X_{F}=\left(F_{J},-F_{\theta},\left\{\mathrm{i} F_{q_{n}}\right\},\left\{-\mathrm{i} F_{\bar{q}_{n}}\right\}\right)$ associated with a function $F$ on $D(\sigma, r) \times \mathcal{O}$, its weighted norm is defined as

$$
\begin{aligned}
\left\|X_{F}\right\|_{D(\sigma, r), \mathcal{O}} \\
\equiv \\
\equiv F_{J}\left\|_{D(\sigma, r), \mathcal{O}}+\frac{1}{r^{2}}\right\| F_{\theta} \|_{D(\sigma, r), \mathcal{O}} \\
\quad+\frac{1}{r}\left(\sum_{n \in \mathbb{Z}^{d}}\left\|F_{q_{n}}\right\|_{D(\sigma, r), \mathcal{O}}|n|^{\bar{a}} e^{|n| \rho}+\sum_{n \in \mathbb{Z}^{d}}\left\|F_{\bar{q}_{n}}\right\|_{D(\sigma, r), \mathcal{O}}|n|^{\bar{a}} e^{|n| \rho}\right) .
\end{aligned}
$$

Lemma 3.1 For $\varepsilon^{*}>0$ sufficiently small and $r=\varepsilon^{*}$, if $|J|<r^{2}$ and $\|q\|_{a, \rho}<r$, then for $\varepsilon=$ $\varepsilon\left(\varepsilon^{*}\right)$, we have

$$
\left\|X_{R}\right\|_{D(\sigma, r), \mathcal{O}}<\varepsilon, \quad \bar{a}=a+1 .
$$

For the proof, see [24].

Now we state our theorem.

Theorem 3.2 Suppose that the Hamiltonian $H$ in (2.5) satisfies assumption (H1)-(H2). Then there exist $\varepsilon>0$ small enough and a Cantor set $\mathcal{O}^{*} \subset \mathcal{O}$ such that meas $\left(\mathcal{O} \backslash \mathcal{O}^{*}\right)=\varepsilon^{\frac{1}{6}}$ and (the smallness condition)

$$
\left\|X_{R}\right\|_{D(\sigma, r), \mathcal{O}}<\varepsilon, \quad \bar{a}=a+1 .
$$


(i) For each $\omega \in \mathcal{O}^{*}$, there exists a real analytic linearly symplectic coordinate transformation

$$
\Sigma^{\infty}: D(\sigma / 2,0) \times \mathcal{O}^{*} \rightarrow D(\sigma, r)
$$

The symplectic coordinate transformation $\Sigma^{\infty}$ is close to the identity:

$$
\left|\Sigma^{\infty}-\mathrm{id}\right|_{D(\sigma / 2,0) \times \mathcal{O}^{*}}^{*}<C \varepsilon^{b}
$$

where $C>0$ is an absolute constant.

(ii) The symplectic coordinate transformation $\Sigma^{\infty}$ transforms the Hamiltonian (2.5) into

$$
H^{\infty}=H \circ \Sigma^{\infty}=\sum_{m=i_{1}}^{\infty} \omega_{m} J_{m}+\sum_{n \in \mathbb{Z}^{d}} \lambda_{n \infty} q_{n} \bar{q}_{n}
$$

where $\omega^{*}=\left(\omega_{i_{1}}, \omega_{i_{2}}, \ldots\right) \in \mathcal{O}^{*}, i_{j} \in \mathcal{I}_{\infty}$, and $\lambda_{n \infty}=|n|^{2}+\mu+\varepsilon^{b_{0}} \frac{\bar{\psi}_{0}^{b_{0}}}{2 \lambda_{n}}+O\left(\varepsilon^{b_{1}}\right)$.

Remark 3.3 The forced term $\psi(\omega t)$ is almost periodic with an infinite-dimensional frequency $\omega=\left(\omega_{1}, \ldots\right)$. A significantly difficult problem is estimating the small divisors at each KAM step because of treating infinite frequencies at the same time. To overcome this difficulty, we split the infinite frequencies to the sum of some finite frequencies, which means that at each KAM step, we only treat some finite frequencies.

Remark 3.4 By the chosen finitely many frequencies at each KAM step the Hamiltonian $H$ in (2.5) needs to be expanded into the proper series of $H=H_{0}^{1}+H_{1}+\cdots+H_{j}+\cdots$. We will transform them in a proper order at the KAM iteration. Thus, in the reducibility, we construct the proper Hamiltonian iteration sequences $\left\{H_{l}^{1}\right\}_{l=0}^{\infty}$ and $\left\{H_{j l}^{3}\right\}_{l=0}^{\infty}, j=l+1, \ldots$.

Remark 3.5 Assumption (H2) is crucial. The KAM iteration is successful because of the new perturbation reducing speedily after each KAM step. The new added perturbation $\varepsilon_{v+1} P_{(v+1)(v+1)}^{3}$ defined in (3.34) at the next KAM step should be smaller than the previous one. The coefficients $\varepsilon^{b_{j}}$ of $\psi(\theta)=\sum_{j=0}^{\infty} \varepsilon^{b_{j}} \psi_{b_{j}}\left(\theta_{1}^{b_{j}}\right)$ will be decided by the estimations of the small divisor measure and the new perturbation in reducibility.

In Sects. 3.2-3.8, we will prove Theorem 3.2 via an improved KAM reducibility.

\subsection{Construction of iterative series and verification of the first KAM iteration}

We iteratively construct Hamiltonian series $\left\{H_{l}^{1}\right\}_{l=0}^{\infty}$ as follows:

$$
H_{l}^{1}=\Lambda_{l}^{1}+\varepsilon_{l} P_{l}^{1}\left(q, \bar{q}, \omega^{b_{l}}, \varepsilon\right), \quad l=0,1, \ldots, \nu,
$$

where

$$
\begin{aligned}
& \Lambda_{l}^{1}=\left\langle\omega^{b_{l}}, J^{b_{l}}\right\rangle+\sum_{n \in \mathbb{Z}^{d}} \lambda_{n l}(\varepsilon) q_{n} \bar{q}_{n}, \\
& P_{l}^{1}=\sum_{\alpha, \beta} P_{l \alpha \beta}^{1}\left(\theta^{b_{l}}\right) q^{\alpha} \bar{q}^{\beta}=\sum_{n, m \in \mathbb{Z}^{d}}\left(P_{n m}^{120 b_{l}}\left(\theta^{b_{l}}\right) q_{n} q_{m}+P_{n m}^{111 b_{l}}\left(\theta^{b_{l}}\right) q_{n} \bar{q}_{m}+P_{n m}^{102 b_{l}}\left(\theta^{b_{l}}\right) \bar{q}_{n} \bar{q}_{m}\right),
\end{aligned}
$$


with $P_{n m}^{120 b_{0}}=R_{0 n m}^{20 b_{0}}, P_{n m}^{111 b_{0}}=R_{0 n m}^{11 b_{0}}, P_{n m}^{102 b_{0}}=R_{0 n m}^{102 b_{0}}$ defined in (2.7) and

$$
\begin{aligned}
& P_{l \alpha \beta}^{1}\left(\theta^{b_{l}}\right)=\sum_{k \in \mathbb{Z}^{b} l} \frac{1}{\sqrt{\lambda^{\alpha+\beta}}} P_{l k \alpha \beta}^{1} e^{\mathrm{i}\left\langle k, \theta^{b_{l}}\right\rangle}, \\
& P_{n m}^{1 m_{1} m_{2} b_{l}}=\sum_{k \in \mathbb{Z}^{b_{l}}} \frac{1}{\sqrt{\lambda_{n} \lambda_{m}}} P_{k n m}^{1 m_{1} m_{2} b_{l}} e^{\mathrm{i}\left\langle k, \theta^{b} l\right\rangle}, \quad\left(m_{1}, m_{2}\right)=\{(2,0),(1,1),(0,2)\} .
\end{aligned}
$$

Moreover,

$$
\begin{aligned}
& P_{l k \alpha \beta}^{1}=0 \quad \text { whenever } \sum_{n \in \mathbb{Z}^{d}}\left(\alpha_{n}-\beta_{n}\right) n \neq 0, \quad l=0,1, \ldots, v, \\
& \left\|P_{l}^{1}\left(q, \bar{q}, \omega^{b_{l}}, \varepsilon\right)\right\|_{\Theta_{l} \times \mathcal{O}^{b} l} \leq C, \quad l=0,1, \ldots, v, \quad \bar{a}=a+1, \\
& \lambda_{n 0}=\lambda_{n}=|n|^{2}+\mu, \quad \lambda_{n l}=\lambda_{n}+\sum_{\tilde{s}=0}^{l-1} \varepsilon_{\tilde{s}} \tilde{\lambda}_{n, \tilde{s}}(\varepsilon), \quad l \geq 1,
\end{aligned}
$$

where

$$
\varepsilon_{0} \tilde{\lambda}_{n, 0}(\varepsilon)=\frac{\varepsilon^{b_{0}} \bar{\psi}_{0}^{b_{0}}}{2 \lambda_{n}}, a \tilde{\lambda}_{n, \tilde{s}}(\varepsilon)=\frac{1}{\lambda_{n}} P_{0 n n}^{111 b_{\tilde{s}}}, \quad \tilde{s}=1, \ldots, l-1
$$

We also need another Hamiltonian series $\left\{H_{j l}^{3}\right\}_{l=0}^{\infty}, j=l+1, \ldots$, of the form

$$
H_{j l}^{3}=\sum_{m=b_{j-1}+1}^{b_{j}} \omega_{i_{m}} J_{i_{m}}+\varepsilon_{j} P_{j l}^{3}\left(q, \bar{q}, \omega_{1}^{b_{j}}, \varepsilon\right), \quad j \geq l+1,
$$

where

$$
P_{j l}^{3}\left(q, \bar{q}, \omega_{1}^{b_{j}}, \varepsilon\right)=\sum_{\alpha, \beta} P_{j l \alpha \beta}^{3}\left(\theta_{1}^{b_{j}}\right) q^{\alpha} \bar{q}^{\beta}=\sum_{n, m \in \mathbb{Z}^{d}}\left(P_{j l n m}^{320} q_{n} q_{m}+P_{j l n m}^{311} q_{n} \bar{q}_{m}+P_{j l n m}^{302} \bar{q}_{n} \bar{q}_{m}\right)
$$

with

$$
\begin{aligned}
& P_{j l \alpha \beta}^{3}\left(\theta_{1}^{b_{j}}\right)=\sum_{k \in \mathbb{Z}^{b_{j}-b_{j-1}}} \frac{1}{\sqrt{\lambda^{\alpha+\beta}}} P_{j l k \alpha \beta}^{3} e^{\mathrm{i}\left\langle k, \theta_{1}^{b_{j}}\right\rangle}, \\
& P_{j l n m}^{3 m_{1} m_{2}}=\sum_{k \in \mathbb{Z}^{b_{j}-b_{j-1}}} \frac{1}{\sqrt{\lambda_{n} \lambda_{m}}} P_{j l k n m}^{3 m_{1} m_{2}} e^{\mathrm{i}\left\langle k, \theta_{1}^{b_{j}}\right\rangle}, \quad\left(m_{1}, m_{2}\right)=\{(2,0),(1,1),(0,2)\} .
\end{aligned}
$$

Moreover,

$$
\begin{aligned}
& P_{j l k \alpha \beta}^{3}=0, \quad \text { whenever } \sum_{n \in \mathbb{Z}^{d}}\left(\alpha_{n}-\beta_{n}\right) n \neq 0, \quad l=0,1, \ldots, v, \\
& \left\|P_{j l}^{3}\left(q, \bar{q}, \omega_{1}^{b_{j}}, \varepsilon\right)\right\|_{\Theta_{j} \times \mathcal{O}^{b_{j}}} \leq C, \quad j \geq l+1, l=0,1, \ldots, v, \quad \bar{a}=a+1 .
\end{aligned}
$$

We easily verify that $H_{0}^{1}=\left.H_{l}^{1}\right|_{l=0}$, that is, $(3.1)_{0}$ is satisfied. It is easy to obtain $\lambda_{n 0}$ by (2.7), that is, (3.4) $)_{0}$ is satisfied. Let $H_{j}=\left.H_{j l}^{3}\right|_{l=0}$, that is, $(3.5)_{0}$, be satisfied. From Assumption (H2) 
and Lemma 3.1 we obtain that

$$
\left\|P_{0}\left(q, \bar{q}, \theta^{b_{l}}, \varepsilon\right)\right\|_{\Theta_{0} \times \mathcal{O}^{b_{0}}} \leq C, \quad\left\|P_{j 0}^{3}\left(q, \bar{q}, \omega_{1}^{b_{j}}, \varepsilon\right)\right\|_{\Theta_{j} \times \mathcal{O}^{b_{j}}} \leq C,
$$

which means that $(3.3)_{0}$ and $(3.7)_{0}$ are satisfied. From (2.9) by $\left.P_{l k \alpha \beta}\right|_{l=0}=R_{0 k \alpha \beta},\left.P_{j l k \alpha \beta}^{3}\right|_{l=0}=$ $R_{j k \alpha \beta}$ we get $(3.2)_{0}$ and $(3.6)_{0}$.

\subsection{Solve the homological equations}

At each step of the KAM iteration, we will meet the small divisors in finding the coordinate transforms. Now we first estimate the measure of the small divisor about the frequency $\omega \in \mathcal{O}$, which will be proved in Appendix.

Lemma 3.6 For $k \in \mathbb{Z}^{b_{s}}, n, m \in \mathbb{Z}^{d}, l=0,1, \ldots, v$, there exist closed subsets

$$
\mathcal{O}_{*}^{b_{l}}=\left\{\omega^{b_{l}}: \omega^{b_{l}}=\left(\omega_{i_{1}}, \ldots, \omega_{i_{j}}, \ldots, \omega_{i_{b_{l}}}\right), i_{j} \in \mathcal{I}_{l}\right\}
$$

such that, for all $\omega^{b_{l}} \in \mathcal{O}_{*}^{b_{l}}$, we have the following inequalities:

$$
\begin{aligned}
& \left|\left\langle k, \omega^{b_{l}}\right\rangle\right| \geq \frac{\varepsilon_{l}^{\frac{1}{4}}}{\left(1+l^{3}\right)(|k|+1)^{b_{l}+2}}, \\
& \left|\left\langle k, \omega^{b_{l}}\right\rangle \pm\left(\lambda_{n l}+\lambda_{m l}\right)\right| \geq \frac{\varepsilon_{l}^{\frac{1}{4}}}{\left(1+l^{3}\right)(|k|+1)^{b_{l}+2}}, \\
& \left|\left\langle k, \omega^{b_{l}}\right\rangle+\left(\lambda_{n l}-\lambda_{m l}\right)\right| \geq \frac{\varepsilon_{l}^{\frac{1}{4}}}{\left(1+l^{3}\right)(|k|+1)^{b_{l}+2}}, \quad|k|+|| n|-| m|| \neq 0,
\end{aligned}
$$

where $\lambda_{n l}$ and $\lambda_{m l}$ are defined in (3.4) .

Moreover, letting

$$
\begin{aligned}
& \mathcal{O}_{v}^{*}=\left\{\omega: \omega=\left(\omega^{b_{v}}, \omega^{\prime}\right)=\left(\omega_{i_{1}}, \ldots, \omega_{i_{b_{v}}}, \omega_{i_{b_{v}+1}}, \ldots\right) \in \mathcal{O}_{*}^{b_{v}} \times \mathcal{O}_{\nu}^{\prime}, i_{j} \in \mathcal{I}_{\nu}\right\}, \\
& \mathcal{O}^{*}=\bigcap_{\nu=0}^{\infty} \mathcal{O}_{v}^{*},
\end{aligned}
$$

we get

$$
\begin{aligned}
\mathcal{O}_{v}^{*} \subseteq \mathcal{O}_{v-1}^{*} \subseteq \cdots & \subseteq \mathcal{O}_{0}^{*} \subseteq \mathcal{O} \\
\operatorname{meas}\left(\mathcal{O}_{v}^{*} \backslash \mathcal{O}_{v+1}^{*}\right) & \leq \frac{2 \varepsilon_{v+1}^{\frac{5}{24}}}{\left(1+(v+1)^{3}\right)^{1-\frac{1}{d}}}, \operatorname{meas}\left(\mathcal{O}_{v} \backslash \mathcal{O}_{v}^{*}\right) \\
& \leq \frac{2 \varepsilon_{v}^{\frac{5}{24}}}{\left(1+v^{3}\right)^{1-\frac{1}{d}}}, \operatorname{meas}\left(\mathcal{O} \backslash \mathcal{O}^{*}\right) \leq C \varepsilon^{\frac{1}{6}},
\end{aligned}
$$

where $C$ is a constant depending on $\mu$ and $\varrho$.

We look for a change of variables $S_{v}$ defined in a domain $D_{v+1}$ by the time-one map $X_{\mathcal{F}_{v}}^{1}$ of the Hamiltonian vector field $X_{\mathcal{F}_{v}}$. Let $X_{\mathcal{F}_{v}}^{t}$ be the time- $t$ map of the flow of the 
Hamiltonian vector field $X_{\mathcal{F}_{v}}$ given by the Hamiltonian

$$
\begin{aligned}
\mathcal{F}_{v} & =\varepsilon_{v} F_{v} \\
& =\varepsilon_{v} \sum_{k \in \mathbb{Z}^{b_{v}}}\left(\sum_{n, m \in \mathbb{Z}^{d}}\left(F_{k n m}^{20 b_{v}} q_{n} q_{m}+F_{k n m}^{02 b_{v}} \bar{q}_{n} \bar{q}_{m}\right)+\sum_{|k|+|| n|-| m|| \neq 0} F_{k n m}^{11 b_{v}} q_{n} \bar{q}_{m}\right) e^{\mathrm{i}\left\langle k, \theta^{b_{v}}\right\rangle} .
\end{aligned}
$$

For $\omega^{b_{v}} \in \mathcal{O}_{*}^{b_{v}}, S_{v}$ transforms system $(3.1)_{v}$ into

$$
\begin{aligned}
H_{v+1}^{2}:= & H_{v}^{1} \circ S_{v} \\
= & \Lambda_{v}^{1}+\varepsilon_{v} P_{v}^{1}+\varepsilon_{v}\left\{\Lambda_{v}^{1}, F_{v}\right\} \\
& +\varepsilon_{v}^{2} \int_{0}^{1}(1-t)\left\{\left\{\Lambda_{v}^{1}, F_{v}\right\}, F_{v}\right\} \circ X_{\mathcal{F}_{v}}^{t} d t+\varepsilon_{v}^{2} \int_{0}^{1}\left\{P_{v}^{1}, F_{v}\right\} \circ X_{\mathcal{F}_{v}}^{t} d t,
\end{aligned}
$$

and for $j \geq v+1$, Hamiltonian (3.5) $v$ is transformed into

$$
\begin{aligned}
H_{j(v+1)}^{3}:=H_{j v}^{3} \circ S_{v}= & \sum_{m=b_{j-1}+1}^{b_{j}} \omega_{i_{m}} J_{i_{m}}+\varepsilon_{v} P_{j v}^{3}+\varepsilon_{v}\left\{\sum_{m=b_{j-1}+1}^{b_{j}} \omega_{i_{m}} J_{i_{m}}, F_{v}\right\} \\
& +\varepsilon_{v}^{2} \int_{0}^{1}(1-t)\left\{\left\{\sum_{m=b_{j-1}+1}^{b_{j}} \omega_{i_{m}} J_{i_{m}}, F_{v}\right\}, F_{v}\right\} \circ X_{\mathcal{F}_{v}}^{t} d t \\
& +\varepsilon_{v}^{2} \int_{0}^{1}\left\{P_{j v}^{3}, F_{v}\right\} \circ X_{\mathcal{F}_{v}}^{t} d t .
\end{aligned}
$$

Now the unknown function $F_{v}$ needs to satisfy the following equation:

$$
P_{v}^{1}+\left\{\Lambda_{v}^{1}, F_{v}\right\}=\sum_{n \in \mathbb{Z}^{d}} \frac{1}{\lambda_{n}} P_{0 n n}^{111 b_{v}} q_{n} \bar{q}_{n}
$$

which is equivalent to

$$
\begin{aligned}
& \left(\left\langle k, \omega^{b v}\right\rangle-\lambda_{n}-\lambda_{m}\right) F_{k n m}^{20 b_{v}}=\mathrm{i} \frac{1}{\sqrt{\lambda_{n} \lambda_{m}}} P_{k n m}^{120 b_{v}}, \\
& \left(\left\langle k, \omega^{b v}\right\rangle+\lambda_{n}+\lambda_{m}\right) F_{k n m}^{02 b_{v}}=\mathrm{i} \frac{1}{\sqrt{\lambda_{n} \lambda_{m}}} P_{k n m}^{102 b_{v}}, \\
& \left(\left\langle k, \omega^{b v}\right\rangle-\lambda_{n}+\lambda_{m}\right) F_{k n m}^{11 b_{v}}=\mathrm{i} \frac{1}{\sqrt{\lambda_{n} \lambda_{m}}} P_{k n m}^{111 b_{v}}, \quad|k|+|| n|-| m|| \neq 0 .
\end{aligned}
$$

Inserting $F$ into (3.8), we have

$$
\begin{aligned}
& F_{n m}^{20 b_{v}}=\sum_{k \in \mathbb{Z}^{b_{v}}} \frac{\mathrm{i} P_{k n n}^{120 b_{v}}}{\sqrt{\lambda_{n} \lambda_{m}}\left(\left\langle k, \omega^{b_{v}}\right\rangle-\left(\lambda_{n v}+\lambda_{m v}\right)\right)} e^{\mathrm{i}\left\langle k, \theta^{b_{v}}\right\rangle}, \\
& F_{n m}^{02 b_{v}}=\sum_{k \in \mathbb{Z}^{b_{v}}} \frac{\mathrm{i} P_{k n m}^{102 b_{v}}}{\sqrt{\lambda_{n} \lambda_{m}}\left(\left\langle k, \omega^{b_{v}}\right\rangle+\left(\lambda_{n v}+\lambda_{m v}\right)\right)} e^{\mathrm{i}\left\langle k, \theta^{b_{v}}\right\rangle}, \\
& F_{n m}^{11 b_{v}}=\sum_{k \in \mathbb{Z}^{b_{v}},|k|+|| n|-| m|| \neq 0} \frac{\mathrm{i} P_{k n m}^{111 b_{v}}}{\left.\sqrt{\lambda_{n} \lambda_{m}}\left(\left\langle k, \omega^{b_{v}}\right\rangle-\lambda_{n v}+\lambda_{m v}\right)\right)} e^{\mathrm{i}\left\langle k, \theta^{b_{v}}\right\rangle} .
\end{aligned}
$$


Since $j \geq v+1$, we obtain $b_{j} \geq b_{v+1}$. From the definition of $F_{v}\left(\theta^{b_{v}}\right)$ in (3.8) and $\theta^{b_{v}}=$ $\left(\theta_{i_{1}}, \ldots, \theta_{i_{b_{v}}}\right)$ we obtain that $\partial_{\theta_{m}} F_{v}=0, m=b_{j-1}+1, \ldots, b_{j}$. Thus

$$
\begin{aligned}
& \left\{\sum_{m=b_{j-1}+1}^{b_{j}} \omega_{i_{m}} J_{i_{m}}, F_{v}\right\} \\
& \quad=\sum_{m=1}^{b_{j}}\left[\partial_{J_{m}}\left(\sum_{s=b_{j-1}+1}^{b_{j}} \omega_{i_{s}} J_{i_{s}}\right)\right]\left[\partial_{\theta_{m}}\left(F_{v}\right)\right] \\
& =\sum_{m=1}^{b_{j-1}}\left[\partial_{J_{m}}\left(\sum_{s=b_{j-1}+1}^{b_{j}} \omega_{i_{s}} J_{i_{s}}\right)\right]\left[\partial_{\theta_{m}}\left(F_{v}\right)\right]+\sum_{m=b_{j-1}+1}^{b_{j}}\left[\partial_{J_{m}}\left(\sum_{s=b_{j-1}+1}^{b_{j}} \omega_{i_{s}} J_{i_{s}}\right)\right]\left[\partial_{\theta_{m}}\left(F_{v}\right)\right] \\
& =0 .
\end{aligned}
$$

Thus (3.10) can be rewritten as

$$
H_{j(v+1)}^{3}=H_{j v}^{3} \circ S_{v}=\sum_{m=b_{j-1}+1}^{b_{j}} \omega_{i_{m}} J_{i_{m}}+\varepsilon_{j} P_{j \nu}^{3}+\varepsilon_{\nu} \varepsilon_{j} \int_{0}^{1}\left\{P_{j v}^{3}, F_{v}\right\} \circ X_{\mathcal{F}_{v}}^{t} d t
$$

\subsection{Estimation on the coordinate transformation}

We proceed to estimate $X_{\mathcal{F}_{v}}$ and $\phi_{\mathcal{F}_{v}}^{1}$.

Lemma 3.7 Let $D_{v}^{i}=D\left(\sigma_{v+1}+\frac{i}{4}\left(\sigma_{v}-\sigma_{v+1}\right), \frac{i}{4} r_{v}\right), 0<i \leq 4$. Then

$$
\left\|X_{\mathcal{F}_{v}}\right\|_{D_{v}^{3}, \mathcal{O}_{*}^{b_{v}}} \leq C \varepsilon_{v}^{\frac{3}{4}}\left(\frac{256\left(b_{v}+4\right)}{e}\right)^{b_{v}+4}\left(\sigma_{v}-\sigma_{v+1}\right)^{-2 b_{v}-\frac{5}{2}}
$$

Proof Recall that by $\omega^{b_{v}} \in \mathcal{O}_{*}^{b_{v}},(3.6)$, and (3.12) we get, for $\left(m_{1}, m_{2}\right)=\{(2,0),(0,2)\}$,

$$
\begin{aligned}
& \sup _{\omega^{b_{v}} \in \mathcal{O}_{*}^{b_{v}}}\left|F_{k n m}^{m_{1} m_{2} b_{v}}\right| \leq C\left|\frac{1}{\sqrt{\lambda_{n} \lambda_{m}}} P_{k n m}^{1 m_{1} m_{2} b_{v}}\right|_{\mathcal{O}_{*}^{b_{v}}} \varepsilon_{v}^{-\frac{1}{4}}\left(1+v^{3}\right)(|k|+1)^{b_{v}+2}, \\
& \sup _{\omega^{b_{v}} \in \mathcal{O}_{*}^{b_{v}}}\left|F_{k n m}^{11 b_{v}}\right| \leq C\left|\frac{1}{\sqrt{\lambda_{n} \lambda_{m}}} P_{k n m}^{111 b_{v}}\right|_{\mathcal{O}_{*}^{b_{v}}} \varepsilon_{v}^{-\frac{1}{4}}\left(1+v^{3}\right)(|k|+1)^{b_{v}+2}, \\
& |k|+|| n|-| m|| \neq 0 .
\end{aligned}
$$

Recalling $(3.4)_{v}$, we get

$$
\left|\partial_{\omega^{b_{v}}} \lambda_{n, v}(\varepsilon)\right| \leq \frac{\varepsilon^{b_{0}}}{\lambda_{n}}
$$

Thus, in view of (3.12), (3.18), and (3.3) $)_{v}$, for $\left(\theta^{b_{\nu}} ; \omega^{b_{v}}\right) \in \Theta_{\nu+1} \times \mathcal{O}_{*}^{b_{v}}$, we deduce that for $|k|+|| n|-| m|| \neq 0$

$$
\left|\partial_{\omega^{b_{\nu}}} F_{k n m}^{11 b_{v}}\right| \leq\left|\frac{\mathrm{i} \partial_{\omega^{b_{v}}} P_{k n m}^{111 b_{v}}}{\sqrt{\lambda_{n} \lambda_{m}}\left(\left\langle k, \omega^{b_{v}}\right\rangle+\lambda_{n v}-\lambda_{m v}\right)}\right|+\left|\frac{\mathrm{i}\left(|k|+C \varepsilon^{b_{0}}\right) P_{k n m}^{111 b_{v}}}{\sqrt{\lambda_{n} \lambda_{m}}\left(\left\langle k, \omega^{b_{v}}\right\rangle+\lambda_{n v}-\lambda_{m v}\right)^{2}}\right|
$$




$$
\leq C\left|\frac{1}{\sqrt{\lambda_{n} \lambda_{m}}} P_{k n m}^{111 b_{\nu}}\right|_{\mathcal{O}_{*}^{b_{\nu}}} \varepsilon_{v}^{-\frac{1}{4}}\left(1+v^{3}\right)(|k|+1)^{b_{\nu}+3} .
$$

Similarly, we get, for $\left(m_{1}, m_{2}\right)=\{(2,0),(0,2)\}$,

$$
\left|\partial_{\omega^{b_{\nu}}} F_{k n m}^{m_{1} m_{2} b_{\nu}}\right| \leq\left|\frac{1}{\sqrt{\lambda_{n} \lambda_{m}}} P_{k n m}^{1 m_{1} m_{2} b_{\nu}}\right|_{\mathcal{O}_{*}^{b_{\nu}}} \varepsilon_{\nu}^{-\frac{1}{4}}\left(1+\nu^{3}\right)(|k|+1)^{b_{\nu}+3} .
$$

Thus by (3.16)-(3.20) we get, for $\left(m_{1}, m_{2}\right)=\{(2,0),(1,1),(0,2)\}$,

$$
\left|F_{k n m}^{m_{1} m_{2} b_{\nu}}\right|_{\mathcal{O}_{*}^{b_{\nu}}} \leq C\left|\frac{1}{\sqrt{\lambda_{n} \lambda_{m}}} P_{k n m}^{1 m_{1} m_{2} b_{v}}\right|_{\mathcal{O}_{*}^{b_{\nu}}} \varepsilon_{v}^{-\frac{1}{4}}\left(1+v^{3}\right)(|k|+1)^{b_{\nu}+3} .
$$

By the definition of the weighted norm, $\sigma_{v+1}+\frac{3}{4}\left(\sigma_{v}-\sigma_{v+1}\right)=\sigma_{v}-\frac{1}{4}\left(\sigma_{v}-\sigma_{v+1}\right),(3.3)_{v}$, and (3.21) it follows that

$$
\begin{aligned}
& \frac{1}{r_{v}^{2}}\left\|\partial_{\theta^{b_{v}}} F_{v}\right\|_{D_{\nu}^{3}, \mathcal{O}_{*}^{b_{v}}} \\
& \leq \frac{1}{r_{v}^{2}} \sum_{|k|+|| n|-| m|| \neq 0}\left|F_{k n m}^{11 b_{v}}\right|_{\mathcal{O}_{*}^{b_{v}}}\left|q_{n}\right|\left|\bar{q}_{m}\right||k| e^{|k|\left(\sigma_{v}-\frac{1}{4}\left(\sigma_{v}-\sigma_{v+1}\right)\right)} \\
& +\frac{1}{r_{v}^{2}} \sum_{k, n, m}\left(\left|F_{k n m}^{20 b_{v}}\right|_{\mathcal{O}_{*}^{b_{v}}}\left|q_{n}\right|\left|q_{m}\right|+\left|F_{k n m}^{02 b_{v}}\right|_{\mathcal{O}_{*}^{b_{v}}}\left|\bar{q}_{n}\right|\left|\bar{q}_{m}\right|\right)|k| e^{|k|\left(\sigma_{v}-\frac{1}{4}\left(\sigma_{\nu}-\sigma_{v+1}\right)\right)} \\
& \leq \frac{1}{r_{v}^{2}} \varepsilon_{v}^{-\frac{1}{4}}\left(1+v^{3}\right)(|k|+1)^{b_{v}+4} e^{\left.-\frac{1}{4}\left(\sigma_{v}-\sigma_{v+1}\right)\right)} \\
& \times\left[\sum_{|k|+|| n|-| m|| \neq 0}\left|\frac{1}{\sqrt{\lambda_{n} \lambda_{m}}} P_{k n m}^{11 b_{v}}\right|_{\mathcal{O}_{*}^{b_{\nu}}}\left|q_{n}\right|\left|\bar{q}_{m}\right| e^{|k| \sigma_{\nu}}\right. \\
& \left.+\sum_{k, n, m}\left(\left|\frac{1}{\sqrt{\lambda_{n} \lambda_{m}}} P_{k n m}^{20 b_{\nu}}\right|_{\mathcal{O}_{*}^{b_{\nu}}}\left|q_{n}\right|\left|q_{m}\right|+\left|\frac{1}{\sqrt{\lambda_{n} \lambda_{m}}} P_{k n m}^{02 b_{\nu}}\right|_{\mathcal{O}_{*}^{b_{\nu}}}\left|\bar{q}_{n}\right|\left|\bar{q}_{m}\right|\right) e^{|k| \sigma_{\nu}}\right] \\
& \leq \varepsilon_{v}^{-\frac{1}{4}}\left(\frac{256\left(b_{v}+4\right)}{e}\right)^{b_{v}+4}\left(\sigma_{v}-\sigma_{v+1}\right)^{-2 b_{v}-\frac{5}{2}}\left\|X_{P_{v}^{1}}\right\|_{D_{v}^{3}, \mathcal{O}_{*}^{b_{v}}} \\
& \leq C \varepsilon_{v}^{-\frac{1}{4}}\left(\frac{256\left(b_{v}+4\right)}{e}\right)^{b_{v}+4}\left(\sigma_{v}-\sigma_{v+1}\right)^{-2 b_{v}-\frac{5}{2}} \text {. }
\end{aligned}
$$

By the definition of $F_{v}$ we easily see that $\partial_{J^{b_{v}}} F_{v}=0$ and

$$
\begin{aligned}
& \left\|\partial_{q_{n}} F_{v}\right\|_{D_{v}^{3}, \mathcal{O}_{*}^{b_{v}}} \\
& \leq\left\|\sum_{k, m} F_{k n m}^{11 b_{v}} \bar{q}_{m} e^{\mathrm{i}<k, \theta^{b_{v}}>}+\sum_{k, m} F_{k n m}^{20 b_{v}} q_{m} e^{\mathrm{i}<k, \theta^{b_{\nu}}>}\right\|_{D_{v}^{3}, \mathcal{O}_{*}^{b_{\nu}}} \\
& \leq \varepsilon_{\nu}^{-\frac{1}{4}}\left(1+v^{3}\right)(|k|+1)^{b_{\nu}+4} \sum_{k, m}\left|\frac{1}{\sqrt{\lambda_{n} \lambda_{m}}} P_{k n m}^{11 b_{\nu}}\right|_{\mathcal{O}_{*}^{b_{\nu}}}\left|\bar{q}_{m}\right| e^{|k|\left(\sigma_{\nu}-\frac{1}{4}\left(\sigma_{\nu}-\sigma_{\nu+1}\right)\right)} \\
& +\varepsilon_{\nu}^{-\frac{1}{4}}\left(1+v^{3}\right)(|k|+1)^{b_{\nu}+4} \sum_{k, m}\left|\frac{1}{\sqrt{\lambda_{n} \lambda_{m}}} P_{k n m}^{20 b_{\nu}}\right|_{\mathcal{O}_{*}^{b_{\nu}}}\left|q_{m}\right| e^{|k|\left(\sigma_{\nu}-\frac{1}{4}\left(\sigma_{\nu}-\sigma_{\nu+1}\right)\right)} .
\end{aligned}
$$


Similarly,

$$
\begin{aligned}
& \left\|\partial_{\bar{q}_{n}} F_{v}\right\|_{D_{v}^{3}, \mathcal{O}_{*}^{b_{v}}} \\
& \leq \varepsilon_{\nu}^{-\frac{1}{4}}\left(1+\nu^{3}\right)(|k|+1)^{b_{v}+4} \sum_{k, m}\left|\frac{1}{\sqrt{\lambda_{n} \lambda_{m}}} P_{k m n}^{11 b_{\nu}}\right|_{\mathcal{O}_{*}^{b_{\nu}}}\left|q_{m}\right| e^{|k|\left(\sigma_{\nu}-\frac{1}{4}\left(\sigma_{\nu}-\sigma_{v+1}\right)\right)} \\
& +\varepsilon_{\nu}^{-\frac{1}{4}}\left(1+v^{3}\right)(|k|+1)^{b_{\nu}+4} \sum_{k, m}\left|\frac{1}{\sqrt{\lambda_{n} \lambda_{m}}} P_{k n m}^{02 b_{v}}\right|_{\mathcal{O}_{*}^{b_{v}}}\left|\bar{q}_{m}\right| e^{|k|\left(\sigma_{\nu}-\frac{1}{4}\left(\sigma_{\nu}-\sigma_{v+1}\right)\right)} .
\end{aligned}
$$

Similarly to the proof of (3.22), by the definition of $\mathcal{F}_{v}$ in (3.8), (3.22)-(3.24), and (3.3) $)_{v}$ we obtain

$$
\begin{aligned}
\left\|X_{\mathcal{F}_{v}}\right\|_{D_{v}^{3}, \mathcal{O}_{*}^{b_{v}}} & \leq \varepsilon_{\nu} \varepsilon_{v}^{-\frac{1}{4}}\left(\frac{256\left(b_{\nu}+4\right)}{e}\right)^{b_{v}+4}\left(\sigma_{\nu}-\sigma_{v+1}\right)^{-2 b_{v}-\frac{5}{2}}\left\|X_{P_{v}^{1}}\right\|_{D_{v}^{3}, \mathcal{O}_{*}^{b_{v}}} \\
& \leq C \varepsilon_{v}^{\frac{3}{4}}\left(\frac{256\left(b_{v}+4\right)}{e}\right)^{b_{v}+4}\left(\sigma_{v}-\sigma_{v+1}\right)^{-2 b_{v}-\frac{5}{2}}
\end{aligned}
$$

Now we give some estimates for $\phi_{\mathcal{F}_{v}}^{t}$. We obtain that our coordinate transformation is well defined by the following formula (3.25). We will use inequality (3.26) to check the convergence of the iteration.

Lemma 3.8 Let $\eta_{v}=\varepsilon^{\frac{1}{8} v b+\frac{1}{12} b}\left(\left(\frac{256\left(b_{\nu}+4\right)}{e}\right)^{b_{\nu}+4}\left(\sigma_{v}-\sigma_{v+1}\right)^{-2 b_{v}-\frac{7}{2}}\right)^{\frac{1}{3}}, D_{v}^{i \eta_{\nu}}=D\left(\sigma_{v+1}+\frac{i}{4}\left(\sigma_{v}-\right.\right.$ $\left.\left.\sigma_{v+1}\right), \frac{i}{4} \eta_{v} r_{v}\right), 0<i \leq 4$. If $\varepsilon_{v} \ll\left(\left(\frac{256\left(b_{v}+4\right)}{e}\right)^{-b_{v}-4}\left(\sigma_{v}-\sigma_{v+1}\right)^{2 b_{v}+\frac{5}{2}}\right)^{\frac{3}{2}}$, then we have

$$
\phi_{\mathcal{F}_{v}}^{t}: D_{v}^{2 \eta_{\nu}} \rightarrow D_{v}^{3 \eta_{\nu}}, \quad-1 \leq t \leq 1 .
$$

Moreover,

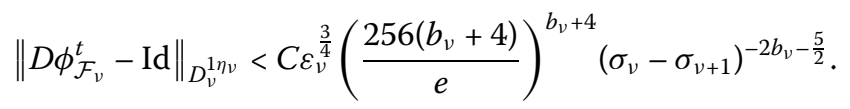

Proof Let

$$
\left|D^{m} \mathcal{F}_{v}\right|_{D_{v}, \mathcal{O}_{*}^{b_{v}}}=\max \left\{\left|\frac{\partial^{|j|+|i|+|\alpha|+|\beta|}}{\partial j^{j} \partial \theta^{i} \partial q^{\alpha} \partial \bar{q}^{\beta}} \mathcal{F}_{v}\right|_{D_{v}, \mathcal{O}_{*}^{b_{v}}},|j|+|i|+|\alpha|+|\beta|=m \geq 2\right\} .
$$

Note that $\mathcal{F}_{v}$ is a polynomial of degree 2 in $q, \bar{q}$. By (3.15), the weighted norm, and the Cauchy inequality we get that for any $m \geq 2$,

$$
\left|D^{m} \mathcal{F}_{v}\right|_{D_{v}^{2}, \mathcal{O}} \leq C \varepsilon_{v}^{\frac{3}{4}}\left(\frac{256\left(b_{v}+4\right)}{e}\right)^{b_{v}+4}\left(\sigma_{v}-\sigma_{v+1}\right)^{-2 b_{v}-\frac{5}{2}}
$$

We consider the integral equation

$$
\phi_{\mathcal{F}_{v}}^{t}=\mathrm{id}+\int_{0}^{t} X_{\mathcal{F}_{v}} \circ \phi_{\mathcal{F}_{v}}^{s} d s
$$

so that $\phi_{\mathcal{F}_{v}}^{t}: D_{v}^{2 \eta_{\nu}} \rightarrow D_{v}^{3 \eta_{\nu}},-1 \leq t \leq 1$, which directly follows from (3.13). Since

$$
D \phi_{\mathcal{F}_{v}}^{t}=\operatorname{Id}+\int_{0}^{t}\left(D X_{\mathcal{F}_{v}}\right) D \phi_{\mathcal{F}_{v}}^{s} d s=\operatorname{Id}+\int_{0}^{t} \mathcal{J}\left(D^{2} \mathcal{F}_{v}\right) D \phi_{\mathcal{F}_{v}}^{s} d s,
$$


where $\mathcal{J}=\left(\begin{array}{cc}0 & -I \\ I & 0\end{array}\right)$, from the Gronwall inequality we get that

$$
\begin{aligned}
\left\|D \phi_{\mathcal{F}_{v}}^{t}-\mathrm{Id}\right\| & \leq\left\|D^{2} \mathcal{F}_{\nu}\right\| e^{\int_{0}^{1}\left\|D^{2} \mathcal{F}_{v}\right\| d s} \leq 2\left\|D^{2} \mathcal{F}_{\nu}\right\| \\
& <C \varepsilon_{\nu}^{\frac{3}{4}}\left(\frac{256\left(b_{v}+4\right)}{e}\right)^{b_{v}+4}\left(\sigma_{v}-\sigma_{v+1}\right)^{-2 b_{v}-\frac{5}{2}} .
\end{aligned}
$$

Consequently, Lemma 3.8 follows.

\subsection{Rewrite the new normal form and new perturbation}

We expand the Hamiltonian $H$ to deal with infinite frequencies $\omega$. Thus we need to rewrite the new Hamiltonian to increase some new finite frequencies in the next iteration. The map $\phi_{\mathcal{F}_{v}}^{1}=S_{v}$ transforms $H_{v}^{1}$ into $H_{v+1}^{2}=H_{v}^{1} \circ \phi_{\mathcal{F}_{v}}^{1}=\Lambda_{v+1}^{2}+\varepsilon_{v+1} P_{v+1}^{2}$. Due to the special form of $P_{v}^{1}$ in (3.2) $)_{v}$, the terms in $\sum_{n m} \frac{1}{\sqrt{\lambda_{n} \lambda_{m}}} P_{0 n m}^{11 b_{v}} q_{n} \bar{q}_{m}$ with $n \neq m$ are absent. Then the new normal form $\Lambda_{v+1}^{2}$ is

$$
\Lambda_{v+1}^{2}=\Lambda_{v}^{1}+\varepsilon_{v} \sum_{n \in \mathbb{Z}^{d}} \frac{1}{\lambda_{n}} P_{0 n n}^{111 b_{v}} q_{n} \bar{q}_{n}=\left\langle\omega^{b_{\nu}}, J^{\nu}\right\rangle+\sum_{n \in \mathbb{Z}^{d}}\left(\lambda_{n v}+\varepsilon_{v} \frac{1}{\lambda_{n}} P_{0 n n}^{111 b_{v}}\right) q_{n} \bar{q}_{n}
$$

We consider the following form of $P_{v+1}^{2}$ :

$$
\begin{aligned}
\varepsilon_{v+1} P_{v+1}^{2} & =\varepsilon_{v}^{2} \int_{0}^{1}(1-t)\left\{\left\{\Lambda_{v}^{1}, F_{v}\right\}, F_{v}\right\} \circ X_{\mathcal{F}_{v}}^{t} d t+\varepsilon_{v}^{2} \int_{0}^{1}\left\{P_{v}^{1}, F_{v}\right\} \circ X_{\mathcal{F}_{v}}^{t} d t \\
& :=\sum_{n, m \in \mathbb{Z}^{d}} \sum_{k \in \mathbb{Z}^{b_{v}}} \frac{\varepsilon_{v}^{2}}{\sqrt{\lambda_{n} \lambda_{m}}}\left(\tilde{P}_{k n m}^{220 b_{v}} q_{n} q_{m}+\tilde{P}_{k n m}^{211 b_{v}} q_{n} \bar{q}_{m}+\tilde{P}_{k n m}^{202 b_{v}} \bar{q}_{n} \bar{q}_{m}\right) e^{\mathrm{i}\left|k, \theta^{b_{\nu}}\right\rangle},
\end{aligned}
$$

where $\tilde{P}_{k n m}^{2 m_{1} m_{2} b_{v}}$ are linear combinations of the products of $F_{k n m}^{m_{1} m_{2} b_{v}}$ and $P_{k n m}^{1 m_{1} m_{2} b_{v}}$. Recalling that $b_{0}=2 b>2, b_{v+1}=b_{v}+b, b_{v} \in \mathbb{Z}^{+}, v=0,1, \ldots, \varepsilon_{v}=\varepsilon^{b_{v}}$, we get

$$
\varepsilon_{v}^{2}=\varepsilon^{2 b_{v}}=\varepsilon_{v+1} \varepsilon^{b+b v}, \quad v=0,1, \ldots
$$

Moreover, we rewrite (3.29) in the form

$$
\begin{aligned}
P_{v+1}^{2}:= & \sum_{n, m \in \mathbb{Z}^{d}, k \in \mathbb{Z}^{b_{\nu}}} \frac{1}{\sqrt{\lambda_{n} \lambda_{m}}}\left(P_{k n m}^{220 b_{v+1}} q_{n} q_{m}+P_{k n m}^{211 b_{\nu+1}} q_{n} \bar{q}_{m}\right. \\
& \left.+P_{k n m}^{202 b_{v+1}} \bar{q}_{n} \bar{q}_{m}\right) e^{\mathrm{i}\left\langle k, \theta^{b_{\nu}}\right\rangle},
\end{aligned}
$$

where

$$
\left|P_{k n m}^{2 m_{1} m_{2} b_{v+1}}\right|=\varepsilon^{b+b v}\left|\tilde{P}_{k n m}^{2 m_{1} m_{2} b_{v}}\right|, \quad\left(m_{1}, m_{2}\right)=\{(2,0),(1,1),(0,2)\} .
$$

Let

$$
\lambda_{n, v+1}(\varepsilon)=\lambda_{n, v}(\varepsilon)+\varepsilon_{v} \tilde{\lambda}_{n, v}(\varepsilon) .
$$

Recalling (3.11), we obtain that

$$
\varepsilon_{v} \tilde{\lambda}_{n, v}(\varepsilon)=\frac{\varepsilon_{v}}{\lambda_{n}} P_{0 n n}^{111 b_{v}}
$$


which means that $\lambda_{n, v+1}(\varepsilon)$ satisfy $(3.4)_{v+1}$. By the regularity of $X_{P_{v}^{1}}$ and Cauchy estimates we have

$$
\left|\lambda_{n, v+1}(\varepsilon)-\lambda_{n, v}(\varepsilon)\right|<\varepsilon_{v}, \quad\left|P_{0 n n}^{111 b_{v}}\right|<C .
$$

Thus we get

$$
H_{v+1}^{2}:=H_{v}^{1} \circ \phi_{\mathcal{F}_{v}}^{1}:=\Lambda_{v+1}^{2}+\varepsilon_{v+1} P_{v+1}^{2}=\left\langle\omega^{b_{\nu}}, J^{b_{\nu}}\right\rangle+\sum_{n \in \mathbb{Z}^{d}} \lambda_{n, v+1}(\varepsilon) q_{n} \bar{q}_{n}+\varepsilon_{v+1} P_{v+1}^{2} .
$$

For $j=v+1, \ldots$, we consider $H_{j(v+1)}^{3}=H_{j v}^{3} \circ S_{v}$ with $H_{j v}^{3}$ defined in (3.5) $v$. From (3.14) we can assume that

$$
H_{j(v+1)}^{3}=H_{j v}^{3} \circ S_{v}=\Lambda_{j v}^{3}+\varepsilon_{j} P_{j(v+1)}^{3}=\Lambda_{j v}^{3}+\varepsilon_{j}\left(P_{j v}^{3}+\varepsilon_{v} \int_{0}^{1}\left\{P_{j v}^{3}, F_{v}\right\} \circ X_{\mathcal{F}_{v}}^{t} d t\right),
$$

where $\Lambda_{j v}^{3}=\sum_{m=b_{j-1}+1}^{b_{j}} \omega_{i_{m}} J_{i_{m}}$, and

$$
\begin{aligned}
P_{j(v+1)}^{3}= & \sum_{n, m \in \mathbb{Z}^{d}} \sum_{k \in \mathbb{Z}^{b_{j}-b_{j-1}}} \frac{1}{\sqrt{\lambda_{n} \lambda_{m}}}\left(P_{j(v+1) k n m}^{320} q_{n} q_{m}+P_{j(v+1) k n m}^{311} q_{n} \bar{q}_{m}\right) e^{\mathrm{i}\left\langle k, \theta_{1}^{b_{j}}\right\rangle} \\
& +\sum_{n, m \in \mathbb{Z}^{d}} \sum_{k \in \mathbb{Z}^{b_{j}-b_{j-1}}} \frac{1}{\sqrt{\lambda_{n} \lambda_{m}}} P_{j(v+1) k n m}^{302} \bar{q}_{n} \bar{q}_{m} e^{\mathrm{i}\left\langle k, \theta_{1} \theta_{j}\right\rangle}
\end{aligned}
$$

where $P_{j(v+1) k n m}^{3 m_{1} m_{2}}$ are linear combinations of the products of $F_{k n m}^{m_{1} m_{2} b_{v}}$ and $P_{j v k n m}^{3 m_{1} m_{2}}$.

From (3.33), (3.34), and the proper expansion of Hamiltonian $H$ in (2.5), we will construct a new Hamiltonian to transform by $S_{v+1}$ at the next KAM step

$$
\begin{aligned}
H_{v+1}^{1} & :=H_{v+1}^{2}+H_{(v+1)(v+1)}^{3} \\
& :=\Lambda_{v+1}^{1}+\varepsilon_{v+1} P_{v+1}^{1}\left(q, \bar{q}, \theta^{b_{v+1}}, \omega^{b_{v+1}}, \varepsilon\right) \\
& =\left\langle\omega^{b_{v+1}}, J^{b_{v+1}}\right\rangle+\sum_{n \in \mathbb{Z}^{d}} \lambda_{n, v+1}(\varepsilon) q_{n} \bar{q}_{n}+\varepsilon_{v+1} P_{v+1}^{2}+\varepsilon_{v+1} P_{(v+1)(v+1)}^{3},
\end{aligned}
$$

where

$$
\begin{aligned}
& \left\langle\omega^{b_{v+1}}, J^{b_{v+1}}\right\rangle=\left\langle\omega^{b_{\nu}}, J^{b_{v}}\right\rangle+\left\langle\omega_{1}^{b_{v+1}}, J_{1}^{b_{v+1}}\right\rangle, \\
& \Lambda_{v+1}^{1}=\left\langle\omega^{b_{v+1}}, J^{b_{v+1}}\right\rangle+\sum_{n \in \mathbb{Z}^{d}} \lambda_{n, v+1}(\varepsilon) q_{n} \bar{q}_{n}, \\
& \varepsilon_{v+1} P_{\nu+1}^{1}\left(q, \bar{q}, \theta^{b_{v+1}}, \omega^{b_{v+1}}\right)=\varepsilon_{v+1} P_{\nu+1}^{2}+\varepsilon_{v+1} P_{(v+1)(v+1)}^{3} .
\end{aligned}
$$

Now we consider the perturbation term in $H \circ S_{0} \circ \cdots \circ S_{v}$. According to (3.33) and (3.34), we get

$$
H^{(v+1)}:=H \circ S_{0} \circ \cdots \circ S_{v}=\left(H_{0}^{1}+\sum_{j=1}^{\infty} H_{j}\right) \circ S_{0} \circ \cdots \circ S_{v}=H_{v+1}^{1}+\sum_{j \geq v+2} H_{j v+1}^{3}
$$




$$
:=\Lambda_{v+1}+P_{v+1}
$$

with the new normal form

$$
\Lambda_{v+1}=\Lambda_{v+1}^{1}+\sum_{j \geq v+2}\left\langle\omega_{1}^{b_{j}}, J_{1}^{b_{j}}\right\rangle=\left\langle\omega^{b_{v+1}}, J^{b_{v+1}}\right\rangle+\sum_{j \geq v+2}\left\langle\omega_{1}^{b_{n}}, J_{1}^{b_{n}}\right\rangle+\sum_{n \in \mathbb{Z}^{d}} \lambda_{n, v+1}(\varepsilon) q_{n} \bar{q}_{n}
$$

and the new perturbation

$$
P_{v+1}=\varepsilon_{v+1} P_{v+1}^{1}\left(q, \bar{q}, \theta^{b_{v+1}}, \omega^{b_{v+1}}\right)+\sum_{j \geq v+2} \varepsilon_{j} P_{j(v+1)}^{3}\left(q, \bar{q}, \theta_{1}^{b_{j}}, \omega_{1}^{b_{j}}\right) .
$$

\subsection{Estimation of the new Hamiltonian}

Firstly, we estimate the small term $P_{v+1}^{2}$. Let $y_{v}(t)=(1-t)\left(\left\{\Lambda_{v}^{1}, \mathcal{F}_{v}\right\}+\varepsilon_{v} P_{v}^{1}\right)+t \varepsilon_{v} P_{v}^{1}$. From (3.11) and (3.29) we have that

$$
\begin{aligned}
\varepsilon_{v+1} P_{v+1}^{2} & =\int_{0}^{1}(1-t)\left\{\left\{\Lambda_{v}^{1}, \mathcal{F}_{v}\right\}, \mathcal{F}_{\nu}\right\} \circ X_{\mathcal{F}_{v}}^{t} d t+\int_{0}^{1}\left\{\varepsilon_{v} P_{v}^{1}, \mathcal{F}_{v}\right\} \circ X_{\mathcal{F}_{v}}^{t} d t \\
& =\int_{0}^{1}\left\{(1-t)\left\{\Lambda_{v}^{1}, \mathcal{F}_{\nu}\right\}+(1-t) \varepsilon_{v} P_{v}^{1}+t \varepsilon_{v} P_{v}^{1}, \mathcal{F}_{v}\right\} \circ X_{\mathcal{F}_{v}}^{t} d t \\
& =\int_{0}^{1}\left\{y_{v}(t), \mathcal{F}_{v}\right\} \circ X_{\mathcal{F}_{v}}^{t} d t .
\end{aligned}
$$

Hence

$$
\varepsilon_{v+1} X_{P_{v+1}^{2}}=\int_{0}^{1}\left(X_{\mathcal{F}_{v}}^{t}\right) * X_{\left\{y_{v}(t), \mathcal{F}_{v}\right\}} d t
$$

Due to the Lemma 7.2 in [33], we obtain

$$
\left\|X_{\left\{y_{v}(t), \mathcal{F}_{v}\right\}}\right\|_{D_{v}^{2 \eta_{v}}} \leq C \eta_{v}^{-2} \varepsilon_{v}^{\frac{7}{4}}\left(\frac{256\left(b_{v}+4\right)}{e}\right)^{b_{v}+4}\left(\sigma_{v}-\sigma_{v+1}\right)^{-2 b_{v}-\frac{7}{2}}
$$

From [13] we have

$$
\left|\left(X_{\mathcal{F}_{\nu}}^{t}\right) * Y\right|_{D_{\nu}^{1 \eta_{\nu}}} \leq|Y|_{D_{v}^{2 \eta_{\nu}}}, \quad 0 \leq t \leq 1
$$

Let $\eta_{v}=\varepsilon^{\frac{1}{8} v b+\frac{1}{12} b}\left(\left(\frac{256\left(b_{v}+4\right)}{e}\right)^{b_{v}+4}\left(\sigma_{v}-\sigma_{v+1}\right)^{-2 b_{v}-\frac{7}{2}}\right)^{\frac{1}{3}} \ll 1$. For $r_{v+1}=\frac{1}{4} \eta_{v} r_{v}$ and $\bar{a}=a+1$, usingf (3.40), (3.41), and (3.42), we get

$$
\begin{aligned}
\varepsilon_{v+1}\left\|X_{P_{v+1}^{2}}\right\|_{D_{v+1}} & \leq C \eta_{v}^{-2} \varepsilon_{v}^{\frac{7}{4}}\left(\frac{256\left(b_{v}+4\right)}{e}\right)^{b_{v}+4}\left(\sigma_{v}-\sigma_{v+1}\right)^{-2 b_{v}-\frac{7}{2}} \\
& \leq C \varepsilon^{\frac{3}{2} v b+\frac{10}{3} b}\left(\left(\frac{256\left(b_{v}+4\right)}{e}\right)^{b_{v}+4}\left(\sigma_{v}-\sigma_{v+1}\right)^{-2 b_{v}-\frac{7}{2}}\right)^{\frac{1}{3}} \\
& \leq C \varepsilon_{v+1} \varepsilon^{\frac{1}{2} v b+\frac{1}{3} b}\left(\left(\frac{256\left(b_{v}+4\right)}{e}\right)^{b_{v}+4}\left(\sigma_{v}-\sigma_{v+1}\right)^{-2 b_{v}-\frac{7}{2}}\right)^{\frac{1}{3}} \\
& \leq C \varepsilon_{v+1} .
\end{aligned}
$$


From (3.34) it follows that

$$
P_{j(v+1)}^{3}=P_{j v}^{3}+\varepsilon_{v} \int_{0}^{1}\left\{P_{j v}^{3}, F_{v}\right\} \circ X_{\mathcal{F}_{v}}^{t} d t, \quad j \geq v+1
$$

Similarly to the previous proof, using $(3.7)_{v}$ we obtain

$$
\varepsilon_{j}\left\|X_{P_{j(v+1)}^{3}}\right\|_{D_{v+1}} \leq C \varepsilon_{j}, \quad j \geq v+1, \quad \bar{a}=a+1 .
$$

By (3.36), (3.43), and (3.44) we estimate the new perturbation at the next KAM iteration:

$$
\varepsilon_{v+1}\left\|P_{v+1}^{1}\right\|_{D_{v+1}} \leq \varepsilon_{v+1}\left\|P_{v+1}^{2}\right\|_{D_{v+1}}+\varepsilon_{v+1}\left\|P_{(v+1)(v+1)}^{3}\right\|_{D_{v+1}} \leq C \varepsilon_{v+1}
$$

By (3.38), (3.44), and (3.45) we estimate the whole perturbation after the $v$ th step of the KAM iteration:

$$
\left\|P_{v+1}\right\|_{D_{v+1}} \leq \varepsilon_{v+1}\left\|P_{v+1}^{1}\right\|_{D_{v+1}}+\sum_{j \geq v+2} \varepsilon_{j}\left\|P_{j(v+1)}^{3}\right\|_{D_{v+1}} \leq C \varepsilon_{v+1} .
$$

\subsection{Verification of (3.3) $)_{v+1}$ and (3.6) $v+1$}

Now we prove that $P_{v+1}^{1}$ satisfies $(3.3)_{v+1}$ and $P_{j(v+1)}^{3}$ satisfies $(3.6)_{v+1}$.

From (3.29), (3.35), and (3.34) it follows that

$$
\begin{aligned}
& \varepsilon_{v+1} P_{v+1}^{1}= \int_{0}^{1}(1-t)\left\{\left\{\Lambda_{v}^{1}, \mathcal{F}_{v}\right\}, \mathcal{F}_{\nu}\right\} \circ X_{\mathcal{F}_{v}}^{t} d t+\int_{0}^{1}\left\{\varepsilon_{v} P_{v}^{1}, \mathcal{F}_{\nu}\right\} \circ X_{\mathcal{F}_{v}}^{t} d t \\
&+\varepsilon_{v+1} P_{(v+1) v}^{3}+\int_{0}^{1}\left\{\varepsilon_{v+1} P_{(v+1) v}^{3}, \mathcal{F}_{v}\right\} \circ X_{\mathcal{F}_{v}}^{t} d t \\
& P_{j(v+1)}^{3}=P_{j v}^{3}+\int_{0}^{1}\left\{P_{j v}^{3}, \mathcal{F}_{v}\right\} \circ X_{\mathcal{F}_{v}}^{t} d t, \quad j=1, \ldots
\end{aligned}
$$

Recall assumption $P_{v}^{1}$ satisfying (3.3) $)_{v}$ and $P_{j v}^{3}$ satisfying $(3.6)_{v}$, we easily see that the normal form at each KAM step has the same form. From the homological equations (3.11) it follows that $\left\{\Lambda_{v}^{1}, \mathcal{F}_{v}\right\}$ has the same form. Thus, to prove that $P_{v+1}^{1}$ satisfies $(3.3)_{v+1}$ and $P_{j(v+1)}^{3}$ satisfies $(3.6)_{v+1}$, we only need to prove that the special form is closed under the Poisson bracket. Now we prove the following lemma.

Lemma 3.9 Suppose that

$$
G(\theta, q, \bar{q})=\sum_{k \alpha_{1} \beta_{1}} G_{k \alpha_{1} \beta_{1}} e^{\mathrm{i}\left\langle k, \theta^{b_{\nu}}\right\rangle} q^{\alpha_{1}} \bar{q}^{\beta_{1}}, \quad F(\theta, q, \bar{q})=\sum_{k^{\prime} \alpha_{2} \beta_{2}} F_{k^{\prime} \alpha_{2} \beta_{2}} e^{\mathrm{i}\left\langle k^{\prime}, \theta^{b_{v}}\right\rangle} q^{\alpha_{2}} \bar{q}^{\beta_{2}}
$$

satisfies

$$
\begin{aligned}
& G_{k \alpha_{1} \beta_{1}}=0 \quad \text { whenever } \sum_{n}\left(\alpha_{1}-\beta_{1}\right) n \neq 0, \\
& F_{k^{\prime} \alpha_{2} \beta_{2}}=0 \quad \text { whenever } \sum_{n}\left(\alpha_{2}-\beta_{2}\right) n \neq 0 .
\end{aligned}
$$


Then

$$
B(\theta, q, \bar{q})=\{G, F\}:=\sum_{k^{\prime \prime} \alpha_{3} \beta_{3}} B_{k^{\prime \prime} \alpha_{3} \beta_{3}} e^{\mathrm{i}\left(k, \theta^{b_{v}}\right)} q^{\alpha_{3}} \bar{q}^{\beta_{3}}
$$

satisfies

$$
B_{k^{\prime \prime} \alpha_{3} \beta_{3}}=0 \quad \text { whenever } \sum_{n}\left(\alpha_{3}-\beta_{3}\right) n \neq 0 .
$$

Proof Let

$$
\begin{aligned}
& G(\theta, q, \bar{q})=\sum_{k, \sum_{n}\left(\alpha_{1 n}-\beta_{1 n}\right) n=0} G_{k \alpha_{1} \beta_{1}} e^{\mathrm{i}\left\langle k, \theta^{b_{\nu}}\right\rangle} q^{\alpha_{1}} \bar{q}^{\beta_{1}}, \\
& F(\theta, q, \bar{q})=\sum_{k^{\prime}, \sum_{n}\left(\alpha_{2 n}-\beta_{2 n}\right) n=0} F_{k^{\prime} \alpha_{2} \beta_{2}} e^{\mathrm{i}\left\langle k^{\prime}, \theta^{b_{\nu}}\right\rangle} q^{\alpha_{2}} \bar{q}^{\beta_{2}} .
\end{aligned}
$$

Since

$$
\begin{aligned}
\{G, F\}= & \mathrm{i} \sum_{n \in \mathbb{Z}^{d}}\left(\frac{\partial G}{\partial q_{n}} \frac{\partial F}{\partial \bar{q}_{n}}-\frac{\partial G}{\partial \bar{q}_{n}} \frac{\partial F}{\partial q_{n}}\right) \\
= & \mathrm{i} \sum_{n \in \mathbb{Z}^{d}} \sum_{A 1} G_{k \alpha_{1} \beta_{1}} F_{k^{\prime} \alpha_{2} \beta_{2}} e^{\mathrm{i}\left\langle k, \theta^{\left.b_{\nu}\right\rangle}\right.} e^{\mathrm{i}\left\langle k^{\prime}, \theta^{\left.b_{\nu}\right\rangle}\right.} q^{\alpha_{1}-e_{n}} \bar{q}^{\beta_{1}} q^{\alpha_{2}} \bar{q}^{\beta_{2}-e_{n}} \\
& -\mathrm{i} \sum_{n \in \mathbb{Z}^{d}} \sum_{A 2} G_{k \alpha_{1} \beta_{1}} F_{k^{\prime} \alpha_{2} \beta_{2}} e^{\mathrm{i}\left\langle k, \theta^{b_{\nu}}\right\rangle} e^{\mathrm{i}\left\langle k^{\prime}, \theta^{\left.b_{\nu}\right\rangle}\right.} q^{\alpha_{1}} \bar{q}^{\beta_{1}-e_{n}} q^{\alpha_{2}-e_{n}} \bar{q}^{\beta_{2}} \\
= & \mathrm{i} \sum_{n \in \mathbb{Z}^{d}} \sum_{A 3} B_{k^{\prime \prime} \alpha_{3} \beta_{3}} e^{\mathrm{i}\left\langle k+k^{\prime}, \theta^{\left.b_{\nu}\right\rangle}\right\rangle} q^{\alpha_{1}+\alpha_{2}-e_{n}} \bar{q}^{\beta_{1}+\beta_{2}-e_{n}},
\end{aligned}
$$

Let $A 1$ denote

$$
\begin{aligned}
& \left(\left(\alpha_{1 n}-1\right)-\beta_{1 n}\right) n+\sum_{m \in \mathbb{Z}^{d} \backslash\{n\}}\left(\alpha_{1 m}-\beta_{1 m}\right) m=-n, \\
& \left(\alpha_{2 n}-\left(\beta_{2 n}-1\right)\right) n+\sum_{m \in \mathbb{Z}^{d} \backslash\{n\}}\left(\alpha_{2 m}-\beta_{2 m}\right) m=n,
\end{aligned}
$$

A2 denote

$$
\begin{aligned}
& \left(\alpha_{1 n}-\left(\beta_{1 n}-1\right)\right) n+\sum_{m \in \mathbb{Z}^{d} \backslash\{n\}}\left(\alpha_{1 m}-\beta_{1 m}\right) m=n, \\
& \left(\left(\alpha_{2 n}-1\right)-\beta_{2 n}\right) n+\sum_{m \in \mathbb{Z}^{d} \backslash\{n\}}\left(\alpha_{2 m}-\beta_{2 m}\right) m=-n,
\end{aligned}
$$

and $A 3$ denote

$$
\left[\left(\alpha_{1 n}+\alpha_{2 n}-1\right)-\left(\beta_{1 n}+\beta_{2 n}-1\right)\right] n+\sum_{m \in \mathbb{Z}^{d} \backslash\{n\}}\left[\left(\alpha_{1 m}+\alpha_{2 m}\right)-\left(\beta_{1 m}+\beta_{2 m}\right)\right] m,
$$


with $k^{\prime \prime}=k+k^{\prime}, \alpha_{3}=\alpha_{1}+\alpha_{2}-e_{n}, \beta_{3}=\beta_{1}+\beta_{2}-e_{n}$. By (3.48) and (3.49) we obtain that (3.48) is equal to

$$
\begin{aligned}
& {\left[\left(\alpha_{1 n}+\alpha_{2 n}-1\right)-\left(\beta_{1 n}+\beta_{2 n}-1\right)\right] n+\sum_{m \in \mathbb{Z}^{d} \backslash\{n\}}\left[\left(\alpha_{1 m}+\alpha_{2 m}\right)-\left(\beta_{1 m}+\beta_{2 m}\right)\right] m} \\
& =\left[\left(\alpha_{1 n}-\left(\beta_{1 n}-1\right)\right) n+\left(\left(\alpha_{2 n}-1\right)-\beta_{2 n}\right) n\right] \\
& \quad+\sum_{m \in \mathbb{Z}^{d} \backslash\{n\}}\left[\left(\alpha_{1 m}-\beta_{1 m}\right) m+\left(\alpha_{2 m}-\beta_{2 m}\right) m\right] \\
& =n+(-n) \\
& =0 .
\end{aligned}
$$

This means that $\{G, F\}$ satisfies (3.47).

The proof of the partial zero-momentum property of the perturbation at each KAM step is obtained by this lemma.

\subsection{Convergence of transformations}

Now we consider the convergence of transformations at the KAM iteration. Firstly, we consider the whole KAM iteration on the reducibility. Recalling (2.5), (3.33), (3.34), and (3.37), we get

$$
\begin{aligned}
& H=H_{0}^{1}+H_{1}+\cdots+H_{j}+\cdots=H_{0}^{1}+\sum_{j=1}^{\infty} H_{j 0}^{3}, \\
& H \circ S_{0}=H_{0}^{1} \circ S_{0}+\sum_{j=1}^{\infty} H_{j} \circ S_{0}=H_{1}^{2}+\sum_{j=1}^{\infty} H_{j 1}^{3}, \\
& H \circ S_{0} \circ S_{1}=\left(H_{1}^{2}+H_{11}^{3}\right) \circ S_{1}+\sum_{j=2}^{\infty} H_{j 1}^{3} \circ S_{1} \\
& \quad=\left(H_{0}^{1} \circ S_{0}+H_{1} \circ S_{0}\right) \circ S_{1}+\sum_{j=2}^{\infty} H_{j} \circ S_{0} \circ S_{1}, \\
& \cdots \quad \begin{array}{l}
\cdots \\
H \circ S_{0} \circ \cdots \circ S_{v}
\end{array}=\left(H_{v}^{2}+H_{v v}^{3}\right) \circ S_{v}+\sum_{j=v+1}^{\infty} H_{j v}^{3} \circ S_{v} \\
& \quad=H_{0}^{1} \circ S_{0} \circ \cdots \circ S_{v}+\sum_{j=1}^{\infty} H_{j} \circ S_{0} \circ \cdots \circ S_{v} .
\end{aligned}
$$

Let $\Sigma^{v+1}:=S_{0} \circ \cdots \circ S_{v}$. Then

$$
H \circ S_{0} \circ \cdots \circ S_{v}=H_{0}^{1} \circ \Sigma^{\nu+1}+\sum_{j=1}^{\infty} H_{j} \circ \Sigma^{v+1} .
$$


Thus we only need to prove the limiting transformation $S_{0} \circ S_{1} \circ \cdots$ converging to a transformation $\Sigma^{\infty}$. Recalling (3.12), we use the KAM iteration inductively:

$$
\Sigma^{v+1}:=S_{0} \circ \cdots \circ S_{v}: D\left(\sigma_{v+1}, r_{v+1}\right) \times \mathcal{O}_{*}^{b_{v+1}} \rightarrow D\left(\sigma_{0}, r_{0}\right) .
$$

For any $\omega \in \mathcal{O}^{*}$ and $M \geq 0$ large enough, we denote

$$
\Sigma^{M}\left(\cdot ; \omega^{b_{M-1}}\right):=S_{0}\left(\cdot ; \omega^{b_{0}}\right) \circ \cdots \circ S_{M-1}\left(\cdot ; \omega^{b_{M-1}}\right): D_{M} \rightarrow D_{0}
$$

as usual, $\Sigma^{0}$ is the identity mapping. From (3.12) we get

$$
\begin{aligned}
\left|D \Sigma^{M}\right|_{D_{M} \times \mathcal{O}^{*}}^{*} & \leq \prod_{s=0}^{M-1}\left|D S_{s}\right|_{D_{s+1} \times \mathcal{O}^{*}}^{*} \\
& \leq \prod_{s \geq 0}\left(1+C \varepsilon_{s}^{\frac{3}{4}}\left(\frac{256\left(b_{s}+4\right)}{e}\right)^{b_{s}+4}\left(\sigma_{s}-\sigma_{s+1}\right)^{-2 b_{s}-\frac{5}{2}}\right) \\
& \leq 2
\end{aligned}
$$

provided that $\varepsilon$ is small enough. Thus we have that

$$
\begin{aligned}
\left|\Sigma^{M+1}-\Sigma^{M}\right|_{D_{M+1} \times \mathcal{O}^{*}}^{*} & \leq\left|D \Sigma^{M}\right|_{D_{M} \times \mathcal{O}^{*}}^{*} \cdot\left|S_{M}-\mathrm{id}\right|_{D_{M+1} \times \mathcal{O}^{*}}^{*} \\
& \leq C \varepsilon_{M}^{\frac{3}{4}}\left(\frac{256\left(b_{M}+4\right)}{e}\right)^{b_{M+4}}\left(\sigma_{M}-\sigma_{M+1}\right)^{-2 b_{M}-\frac{5}{2}} \\
& \leq C \varepsilon^{\frac{M b}{2}+b} .
\end{aligned}
$$

This means that the sequence $\left\{\Sigma^{M}\right\}$ converges uniformly in $D_{M}$ to an analytic map

$$
\Sigma^{\infty}: D(\sigma / 2,0) \rightarrow D(\sigma, r)
$$

So (i) in this theorem is obtained.

Recalling that $\mathcal{O}^{*}=\bigcap_{v=0}^{\infty} \mathcal{O}_{v}^{*}$ and meas $\left(\mathcal{O} \backslash \mathcal{O}^{*}\right) \leq C \varepsilon^{\frac{1}{6}}$, we get a countable infinite sequence of nonresonance frequencies $\omega^{*}=\left(\omega_{i_{1}}, \omega_{i_{2}}, \ldots\right) \in \mathcal{O}^{*}\left(i_{j} \in \mathcal{I}_{\infty}\right)$ of $\psi(\theta)$ close to the original frequencies $\omega=\left(\omega_{1}, \ldots\right)$.

Because the Hamiltonian $H$ in (2.5) satisfies (3.1) ${ }_{v}$ and (3.5) ${ }_{v}$ with $v=0$, the above iterative procedure can run repeatedly. Inductively, it follows that

$$
H^{\infty}:=H \circ \Sigma^{\infty}:=\left\langle\omega^{*}, J^{*}\right\rangle+\sum_{n \in \mathbb{Z}^{d}} \lambda_{n \infty} q_{n} \bar{q}_{n}
$$

where $\omega^{*}=\left(\omega_{i_{1}}, \omega_{i_{2}}, \ldots\right) \in \mathcal{O}^{*}, i_{j} \in \mathcal{I}_{\infty}$

$$
\lambda_{n \infty}=|n|^{2}+\mu+\varepsilon^{b_{0}} \frac{\bar{\psi}_{0}^{b_{0}}}{2 \lambda_{n}}+O\left(\varepsilon^{b_{1}}\right)=|n|^{2}+\mu+O\left(\varepsilon^{b_{0}}\right)\left(\tilde{c}_{n}+\tilde{r}_{n}\left(\varepsilon^{b}\right)\right)
$$

where $\tilde{c}_{n}$ are constants, and $\left|\tilde{r}_{n}\left(\varepsilon^{b}\right)\right| \rightarrow 0$ as $\varepsilon \rightarrow 0$. So (ii) in this theorem is obtained. This completes the proof. 


\section{Appendix}

Now we show the small divisors lemma applied in the proof of the Theorem 3.2.

Proof Lemma 3.6 From the (3.1) $)_{l}$ and (3.4) $l$ we get

$$
\begin{aligned}
& \omega^{b_{l}}=\left(\omega_{i_{1}}, \ldots, \omega_{i_{b_{l}}}\right), \\
& \lambda_{n 0}=\lambda_{n}=|n|^{2}+\mu, \quad \lambda_{n l}=|n|^{2}+\mu+\frac{\varepsilon^{b_{0}} \bar{\psi}_{0}^{b_{0}}}{2 \lambda_{n}}+O\left(\varepsilon_{1}\right), \quad l \geq 1 .
\end{aligned}
$$

Let

$$
\begin{array}{ll}
g_{2}^{l}\left(\omega^{b_{l}}\right)=\left\langle k, \omega^{b_{l}}\right\rangle-\left(\lambda_{n l}-\lambda_{m l}\right), & |k|+|| n|-| m|| \neq 0, \\
g_{1}^{l}\left(\omega^{b_{l}}\right)=\left\langle k, \omega^{b_{l}}\right\rangle \pm\left(\lambda_{n l}+\lambda_{m l}\right), & g_{1}^{0}\left(\omega^{b_{l}}\right)=\left\langle k, \omega^{b_{l}}\right\rangle
\end{array}
$$

and

$$
\begin{aligned}
& \mathcal{R}_{k l}^{0}:=\left\{\omega^{b_{l}}:\left|\left\langle k, \omega^{b_{l}}\right\rangle\right|<\frac{\varepsilon_{l}^{\frac{1}{4}}}{\left(1+l^{3}\right)(|k|+1)^{b_{l}+2}},\right\}, \\
& \mathcal{R}_{k n m l}^{1}:=\left\{\omega^{b_{l}}:\left|\left\langle k, \omega^{b_{l}}\right\rangle \pm\left(\lambda_{n l}+\lambda_{m l}\right)\right|<\frac{\varepsilon_{l}^{\frac{1}{4}}}{\left(1+l^{3}\right)(|k|+1)^{b_{l}+2}},\right\}, \\
& \mathcal{R}_{k n m l}^{2}:=\left\{\omega^{b_{l}}:\left|\left\langle k, \omega^{b_{l}}\right\rangle-\left(\lambda_{n l}-\lambda_{m l}\right)\right|<\frac{\varepsilon_{l}^{\frac{1}{4}}}{\left(1+l^{3}\right)(|k|+1)^{b_{l}+2}},|k|+|| n|-| m|| \neq 0\right\} .
\end{aligned}
$$

We can choose a vector $v^{b_{l}}$ satisfying $\left\langle k, \omega^{b_{l}}\right\rangle=|k|$. Thus we have that

$$
\begin{aligned}
& \left|\frac{d g_{1}^{0}\left(\omega^{b_{l}}+t v^{b_{l}}\right)}{d t}\right| \geq\left|\left\langle k, v^{b_{l}}\right\rangle \geq \frac{1}{3}\right| k \mid, \\
& \left|\frac{d g_{1}^{l}\left(\omega^{b_{l}}+t v^{b_{l}}\right)}{d t}\right| \geq\left|\left\langle k, v^{b_{l}}\right\rangle\right|-\left|O\left(\varepsilon_{1}\right)\right| \geq \frac{1}{3}|k|, \\
& \left|\frac{d g_{2}^{l}\left(\omega^{b_{l}}+t v^{b_{l}}\right)}{d t}\right| \geq\left|\left\langle k, v^{b_{l}}\right\rangle\right|-\left|O\left(\varepsilon_{1}\right)\right| \geq \frac{1}{3}|k|, \quad|k|+|| n|-| m|| \neq 0,
\end{aligned}
$$

provided that $\varepsilon$ is small enough. From (1.4) for arbitrary fixed $\varrho \in(0,1)$, we get $\omega_{i j} \in[\varrho, 2 \varrho]$, $i_{j} \in \mathcal{I}_{l}$. By the Fubini theorem we have that

$$
\begin{aligned}
& \operatorname{meas} \mathcal{R}_{k l}^{0} \leq 6 \varrho^{b_{l}-1} \frac{\varepsilon_{l}^{\frac{1}{4}}}{\left(1+l^{3}\right)(|k|+1)^{b_{l}+3}}, \\
& \operatorname{meas} \mathcal{R}_{k n m l}^{1} \leq 6 \varrho^{b_{l}-1} \frac{\varepsilon_{l}^{\frac{1}{4}}}{\left(1+l^{3}\right)(|k|+1)^{b_{l}+3}}, \\
& \operatorname{meas} \mathcal{R}_{k n m l}^{2} \leq 6 \varrho^{b_{l}-1} \frac{\varepsilon_{l}^{\frac{1}{4}}}{\left(1+l^{3}\right)(|k|+1)^{b_{l}+3}}, \quad|k|+|| n|-| m|| \neq 0 .
\end{aligned}
$$


Let $\mathcal{R}_{0}^{l}=\bigcup_{k \in \mathbb{Z}^{b} l} \mathcal{R}_{k l}^{1}$ and $\mathcal{R}_{1}^{l}=\bigcup_{k \in \mathbb{Z}^{b} l, n, m \in \mathbb{Z}^{d}} \mathcal{R}_{k n m l}^{1}$. Similarly to the proof in Appendix in [32], we obtain

$$
\text { meas } \mathcal{R}_{1}^{0} \leq \frac{\varepsilon_{l}^{\frac{5}{24}}}{1+l^{3}}, \quad \text { meas } \mathcal{R}_{1}^{l} \leq \frac{\varepsilon_{l}^{\frac{5}{24}}}{1+l^{3}} .
$$

Thus we consider $g_{2}^{l}\left(\omega^{b_{l}}\right)=\left\langle k, \omega^{b_{l}}\right\rangle-\left(\lambda_{n l}-\lambda_{m l}\right),|k|+|| n|-| m|| \neq 0$.

Case 1. If $|k| \neq 0$ and ||$n|-| m||=0$, then $g_{2}^{l}\left(\omega^{b_{l}}\right)=\left\langle k, \omega^{b_{l}}\right\rangle$. By the definition of $\mathcal{O}$ in (1.4) we obtain that $\mathcal{R}_{\text {knml }}^{2}$ is empty.

Case 2. We consider ||$n|-| m|| \neq 0$. Without loss of generality, we suppose that $|n|^{2}-$ $|m|^{2}=a \geq 1$. Then there exists $\delta>0$ such that

$$
\left|\lambda_{n l}-\lambda_{m l}-a\right| \leq C\left|\varepsilon^{b_{0}} \bar{\psi}_{0}^{b_{0}}\right|\left|\frac{1}{\lambda_{n}}-\frac{1}{\lambda_{m}}\right|+O\left(\varepsilon_{1}\right) \leq \mathcal{O}\left(\varepsilon_{l}^{\frac{1}{6}}|m|^{-\delta}\right),
$$

Case 2.1. We may suppose $|n|^{2}-|m|^{2}=a \geq|k|\left|\omega^{b_{l}}\right|+1$. Then

$$
\begin{aligned}
\left|g_{2}^{l}\left(\omega^{b_{l}}\right)\right| & =\left|\left\langle k, \omega^{b_{l}}\right\rangle-\left(\lambda_{n l}-\lambda_{m l}\right)\right|=\left.\left|\left\langle k, \omega^{b_{l}}\right\rangle-\right| n\right|^{2}-\mu+|m|^{2}+\mu+O\left(\varepsilon_{0}\right) \mid \\
& \geq a-\left(\left|\left\langle k, \omega^{b_{l}}\right\rangle\right|+\left|O\left(\varepsilon_{0}\right)\right|\right) \geq 1-\left|O\left(\varepsilon_{0}\right)\right| \\
& >\frac{\varepsilon_{l}^{\frac{1}{4}}}{\left(1+l^{3}\right)(|k|+1)^{b_{l}+2}} .
\end{aligned}
$$

This means that $\mathcal{R}_{\text {knml }}^{2}$ is empty.

Case 2.2. If $a<|k|\left|\omega^{b_{l}}\right|+1$, then we have

$$
\left.\mathcal{R}_{\text {knml }}^{2} \subseteq \mathcal{R}_{\text {kam }}^{2}:=\left\{\omega^{b_{l}}:|| k, \omega^{b_{l}}\right\rangle+a \mid \leq \frac{\varepsilon_{l}^{\frac{1}{4}}}{\left(1+l^{3}\right)(|k|+1)^{b_{l}+2}}+O\left(\varepsilon_{l}^{\frac{1}{6}}|m|^{-\delta}\right)\right\}
$$

Moreover, $\mathcal{R}_{\text {knml }}^{2} \subseteq \mathcal{R}_{\text {kam }}^{2} \subseteq \mathcal{R}_{\text {kam } 0}^{2}$ for $|m| \geq\left|m_{0}\right|$.

Now we estimate $\mathcal{R}_{\text {kam }}^{2}$. We choose a vector $v^{b_{l}}$ satisfying $\left\langle k, v^{b_{l}}\right\rangle=|k|$. It follows that

$$
\frac{d\left(\left\langle k, \omega^{b_{l}}+t \nu^{b_{l}}\right\rangle+a\right)}{d t}=\left\langle k, v^{b_{l}}\right\rangle=|k|>0 .
$$

By the Fubini theorem, since $\omega_{i_{j}} \in[\varrho, 2 \varrho], i_{j} \in \mathcal{I}_{l}$, we get that

$$
\text { meas } \mathcal{R}_{\text {kam }_{0}}^{2} \leq \frac{\varepsilon_{l}^{\frac{1}{4}}}{\left(1+l^{3}\right)(|k|+1)^{b_{l}+3}}+\frac{1}{|k|+1} O\left(\varepsilon_{l}^{\frac{1}{6}}\left|m_{0}\right|^{-\delta}\right) .
$$

Let $\mathcal{R}_{2}^{l}=\bigcup_{k \in \mathbb{Z}^{b} l, n, m \in \mathbb{Z}^{d},|n|^{2}-|m|^{2}=a} \mathcal{R}_{k n m l}^{2}$. We get that

$$
\begin{aligned}
& \operatorname{meas} \mathcal{R}_{2}^{l}=\operatorname{meas}\left(\bigcup_{k \in \mathbb{Z}^{b} l} \bigcup_{n, m \in \mathbb{Z}^{d},|n|^{2}-|m|^{2}=a, a<|k| \mid \omega^{b_{l} \mid+1}} \mathcal{R}_{k n m l}^{2}\right)
\end{aligned}
$$

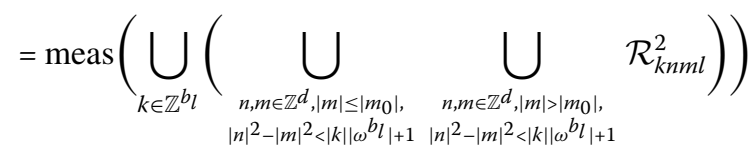




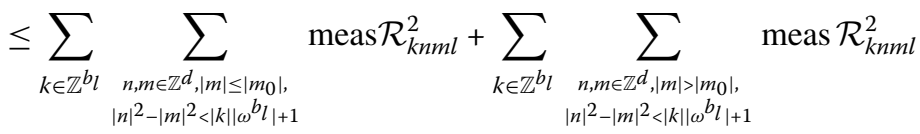

$$
\begin{aligned}
& \leq \sum_{k \in \mathbb{Z}^{b_{l}} \mid}\left(\sum_{\substack{n, m \in \mathbb{Z}^{d},|m| \leq\left|m_{0}\right|,|n|^{2}-|m|^{2}<|k|\left|\omega^{b}\right|+1}} \operatorname{meas} \mathcal{R}_{k n m l}^{2}+\sum_{\substack{n, m \in \mathbb{Z}^{d}, a<|k|\left|\omega^{b} l\right|+1}} \text { meas } \mathcal{R}_{k a m_{0}}^{2}\right) \\
& \leq \sum_{k \in \mathbb{Z}^{b}}\left(|k|\left|\omega^{b_{l}}\right|+1\right)\left(\frac{\left|m_{0}\right|^{d} \varepsilon_{l}^{\frac{1}{4}}}{\left(1+l^{3}\right)(|k|+1)^{b_{l}+3}}+\frac{1}{|k|+1} O\left(\varepsilon_{l}^{\frac{1}{6}}\left|m_{0}\right|^{-\delta}\right)\right) \\
& \leq \sum_{k \in \mathbb{Z}^{b_{l}}}\left(\frac{\left|m_{0}\right|^{d} \varepsilon_{l}^{\frac{1}{4}}}{\left(1+l^{3}\right)(|k|+1)^{b_{l}+2}}+O\left(\varepsilon_{l}^{\frac{1}{6}}\left|m_{0}\right|^{-\delta}\right)\right) \text {. }
\end{aligned}
$$

We choose $\frac{\left|m_{0}\right|^{d} \varepsilon_{l}^{\frac{1}{4}}}{\left(1+l^{3}\right)(|k|+1)^{b_{l}+2}}=\varepsilon_{l}^{\frac{1}{6}}\left|m_{0}\right|^{-\delta}$, that is,

$$
m_{0}=\left(\frac{\left(1+l^{3}\right)(|k|+1)^{b_{l}+2}}{\varepsilon_{l}^{\frac{1}{12}}}\right)^{\frac{1}{d+\delta}} .
$$

Since $\delta>0$ and $d \geq 2$, we have

$$
\frac{1}{4}-\frac{1}{12} \frac{1}{d+\delta} \geq \frac{5}{24}, \quad 1-\frac{1}{d+\delta} \geq 1-\frac{1}{d} .
$$

Thus it follows that

$$
\begin{aligned}
\operatorname{meas} \mathcal{R}_{2}^{l} & \leq \sum_{k \in \mathbb{Z}^{b_{l}}}\left(\frac{\varepsilon_{l}^{\frac{1}{4}-\frac{1}{12} \frac{1}{d+\delta}}}{\left(\left(1+l^{3}\right)(|k|+1)^{b_{l}+2}\right)^{1-\frac{1}{d+\delta}}}\right) \\
& \leq \sum_{k \in \mathbb{Z}^{b_{l}}}\left(\frac{\varepsilon_{l}^{\frac{5}{24}}}{\left(\left(1+l^{3}\right)(|k|+1)^{b_{l}+2}\right)^{1-\frac{1}{d}}}\right) \\
& \leq \frac{\varepsilon_{l}^{\frac{5}{24}}}{\left(1+l^{3}\right)^{1-\frac{1}{d}}}
\end{aligned}
$$

by the convergence of $\sum_{k \in \mathbb{Z}^{b l}} \frac{1}{\left((|k|+1)^{b_{l}+2}\right)^{1-\frac{1}{d}}}$.

Letting $\mathcal{R}^{l}=\mathcal{R}_{0}^{l} \cup \mathcal{R}_{1}^{l} \cup \mathcal{R}_{2}^{l}, \mathcal{R}=\bigcup_{l \geq 0} \mathcal{R}^{l}$, we get

$$
\begin{aligned}
\operatorname{meas} \mathcal{R} & \leq \operatorname{meas}\left(\bigcup_{l \geq 0} \mathcal{R}_{1}^{0}\right) \text { meas }+\left(\bigcup_{l \geq 0} \mathcal{R}_{1}^{l}\right)+\operatorname{meas}\left(\bigcup_{l \geq 0} \mathcal{R}_{2}^{l}\right) \\
& \leq \sum_{l=0}^{\infty}\left(\frac{2 \varepsilon_{l}^{\frac{5}{24}}}{1+l^{3}}+\frac{\varepsilon_{l}^{\frac{5}{24}}}{\left(1+l^{3}\right)^{1-\frac{1}{d}}}\right) \leq \sum_{l=0}^{\infty} \frac{2 \varepsilon_{l}^{\frac{5}{24}}}{\left(1+l^{3}\right)^{1-\frac{1}{d}}} \\
& \leq \varepsilon_{0}^{\frac{1}{6}}
\end{aligned}
$$

by the convergence of $\sum_{l=0}^{\infty} \frac{2}{\left(1+l^{3}\right)^{1-\frac{1}{d}}}$. 
By (3.7) we get that

$$
\begin{aligned}
& \operatorname{meas}\left(\mathcal{O}_{v}^{*} \backslash \mathcal{O}_{v+1}^{*}\right) \leq \operatorname{meas} \mathcal{R}^{v+1} \leq \frac{2 \varepsilon_{v+1}^{\frac{5}{24}}}{\left(1+(v+1)^{3}\right)^{1-\frac{1}{d}}} \\
& \operatorname{meas}\left(\mathcal{O}_{v} \backslash \mathcal{O}_{v}^{*}\right) \leq \operatorname{meas} \mathcal{R}^{v} \leq \frac{2 \varepsilon_{v}^{\frac{5}{24}}}{\left(1+v^{3}\right)^{1-\frac{1}{d}}}
\end{aligned}
$$

From (3.7) and $\varepsilon_{0}=\varepsilon$ we get

$$
\operatorname{meas}\left(\mathcal{O} \backslash \mathcal{O}^{*}\right) \leq \operatorname{meas}\left(\bigcup_{l \geq 0} \mathcal{R}^{l}\right) \leq C \varepsilon^{\frac{1}{6}}
$$

\section{Acknowledgements}

We appreciate the editor and referees for insightful comments and wise advices.

\section{Funding}

This paper is supported by the National Natural Foundation of China (No. 11601270, 11701567).

\section{Abbreviations}

Not applicable.

Availability of data and materials

Data sharing not applicable to this paper as no datasets were generated or analyzed during the current study.

\section{Competing interests}

The authors declare that they have no competing interests.

\section{Authors' contributions}

All authors contributed equally to writing this paper. All authors read and approved the final manuscript.

\section{Author details}

${ }^{1}$ College of Science, China University of Petroleum, Qingdao, People's Republic of China. ${ }^{2}$ School of Mathematics and Quantitative Economics, Shandong University of Finance and Economics, Jinan, People's Republic of China.

\section{Publisher's Note}

Springer Nature remains neutral with regard to jurisdictional claims in published maps and institutional affiliations.

Received: 28 January 2020 Accepted: 2 April 2020 Published online: 10 April 2020

\section{References}

1. Papageorgiou, N.S., Rădulescu, V.D., Repovš, D.D.: Nonlinear Analysis - Theory and Methods. Springer Monogr. Math. Springer, Cham (2019)

2. Xu, R.Z., Wang, X.C., Yang, Y.B., Chen, S.H.: Global solutions and finite time blow-up for fourth order nonlinear damped wave equation. J. Math. Phys. 59(6), 1-27 (2018)

3. Lian, W., Xu, R.Z., Rădulescu, V.D., Yang, Y.B., Zhao, N.: Global well-posedness for a class of fourth order nonlinear strongly damped wave equations. Adv. Calc. Var. (2020). https://doi.org/10.1515/acv-2019-0039

4. Lian, W., Xu, R.Z.: Global well-posedness of nonlinear wave equation with weak and strong damping terms and logarithmic source term. Adv. Nonlinear Anal. 9(1), 613-632 (2020)

5. Mohammed, A., Rădulescu, V.D., Vitolo, A.: Blow-up solutions for fully nonlinear equations: existence, asymptotic estimates and uniqueness. Adv. Nonlinear Anal. 9(1), 39-64 (2020)

6. Papageorgiou, N.S., Rădulescu, V.D., Repovš, D.D.: (p, 2)-equations asymmetric at both zero and infinity. Adv. Nonlinear Anal. 7, 327-351 (2018)

7. Saanouni, T.: Global well-posedness of some high-order focusing semilinear evolution equations with exponential nonlinearity. Adv. Nonlinear Anal. 7(1), 67-84 (2018)

8. Nguyen, H.M.: Superlensing using complementary media and reflecting complementary media for electromagnetic waves. Adv. Nonlinear Anal. 7(4), 449-467 (2018)

9. Goubet, O., Manoubi, I.: Theoretical analysis of a water wave model with a nonlocal viscous dispersive term using the diffusive approach. Adv. Nonlinear Anal. 8(1), 253-266 (2019)

10. Bahrouni, A., Rădulescu, V.D., Repovš, D.D.: Double phase transonic flow problems with variable growth: nonlinear patterns and stationary waves. Nonlinearity 32(7), 2481-2495 (2019)

11. Simon, B.: Tosio Kato's work on non-relativistic quantum mechanics, part 2. Bull. Math. Sci. 9(1), 1950005 (2019)

12. Kuksin, S.B.: Nearly Integrable Infinite-Dimensional Hamiltonian Systems. Lecture Notes in Math., vol. 1556. Springer, New York (1993) 
13. Pöschel, J.: A KAM-theorem for some nonlinear PDEs. Ann. Sc. Norm. Super. Pisa, Cl. Sci. (4) 23(15), 119-148 (1996)

14. Pöschel, J.: Small divisors with spatial structure in infinite-dimensional Hamiltonian systems. Commun. Math. Phys. 127(2), 351-393 (1990)

15. Yuan, X.: Quasi-periodic solutions of completely resonant nonlinear wave equations. J. Differ. Equ. 230, 213-274 (2005)

16. Si, J.: Quasi-periodic solutions of a non-autonomous wave equations with quasi-periodic forcing. J. Differ. Equ. 252, 5274-5360 (2012)

17. Lou, Z.W., Si, J.G.: Quasi-periodic solutions for the reversible derivative nonlinear Schrodinger equations with periodic boundary conditions. J. Dyn. Differ. Equ. 29, 1031-1069 (2017)

18. Bourgain, J.: Construction of approximative and almost-periodic solutions of perturbed linear Schrödinger and wave equations. Geom. Funct. Anal. 6, 201-230 (1996)

19. Pöschel, J.: On the construction of almost-periodic solutions for a nonlinear Schrödinger equation. Ergod. Theory Dyn. Syst. 22, 1537-1549 (2002)

20. Geng, J.S., Xu, X.: Almost-periodic solutions of one dimensional Schrödinger equation with the external parameters. J. Dyn. Differ. Equ. 25, 435-450 (2013)

21. Gao, M.N., Liu, J.J.: Invariant Cantor manifolds of quasi-periodic solutions for the derivative nonlinear Schrödinger equation. J. Differ. Equ. 267, 1322-1375 (2019)

22. Bourgain, J.: Quasi-periodic solutions of Hamiltonian perturbations of $2 \mathrm{D}$ linear Schrödinger equations. Ann. Math. $148,363-439(1998)$

23. Geng, J.S., You, J.G.: KAM tori for higher-dimensional beam equations with constant potentials. Nonlinearity 19 2405-2423 (2006)

24. Geng, J.S., You, J.G.: A KAM theorem for Hamiltonian partial differential equations in higher dimensional spaces. Commun. Math. Phys. 262, 343-347 (2006)

25. Yuan, X.P.: A KAM theorem with applications to partial differential equations of higher dimensions. Commun. Math. Phys. 275, 97-137 (2007)

26. Eliasson, L.H., Kuksin, S.B.: On reducibility of Schrödinger equations with quasiperiodic in time potentials. Commun. Math. Phys. 286, 125-135 (2009)

27. Eliasson, L.H., Kuksin, S.B.: KAM for the nonlinear Schrödinger equations. Ann. Math. 172, 371-435 (2010)

28. Eliasson, L.H., Grébert, B., Kuksin, S.B.: KAM for the nonlinear beam equation. Geom. Funct. Anal. 26, 1588-1715 (2016)

29. Rui, J., Liu, B.C.: Reducibility of beam equations in higher-dimensional spaces. Bound. Value Probl. 2017, 82 (2017)

30. Xu, J.X., You, J.G.: Reducibility of linear differential equations with almost-periodic coefficients. Chin. Ann. Math., Ser. A 17(5), 607-616 (1996) (Chinese)

31. Rui, J., Liu, B.C., Zhang, J.: Almost-periodic solutions for a class of linear Schrödinger equations with almost-periodic forcing. J. Math. Phys. 57, 1-26 (2016)

32. Rui, J., Liu, B.C.: Almost-periodic solutions of an almost-periodically forced wave equation. J. Math. Anal. Appl. 451(2), 629-658 (2017)

33. Niu, H.W., Geng, J.S.: Almost periodic solutions for a class of higher-dimensional beam equations. Nonlinearity 20 2499-2517 (2007)

\section{Submit your manuscript to a SpringerOpen ${ }^{\circ}$ journal and benefit from:}

- Convenient online submission

- Rigorous peer review

Open access: articles freely available online

- High visibility within the field

- Retaining the copyright to your article

Submit your next manuscript at $>$ springeropen.com 